\title{
A closure for Lagrangian velocity gradient evolution in turbulence using recent deformation mapping of initially Gaussian fields
}

\author{
Perry L. Johnson $\dagger$, and Charles Meneveau \\ Department of Mechanical Engineering and Center for Environmental and Applied Fluid \\ Mechanics, The Johns Hopkins University, Baltimore, MD 21218, USA
}

(Received $\mathrm{xx}$; revised $\mathrm{xx}$; accepted $\mathrm{xx}$ )

\begin{abstract}
The statistics of the velocity gradient tensor in turbulent flows are of both theoretical and practical importance. The Lagrangian view provides a privileged perspective for studying the dynamics of turbulence in general, and of the velocity gradient tensor in particular. Stochastic models for the Lagrangian evolution of velocity gradients in isotropic turbulence, with closure models for the pressure Hesssian and viscous Laplacian, have been shown to reproduce important features such as non-Gaussian probability distributions, skewness and vorticity strain-rate alignments. The Recent Fluid Deformation (RFD) closure introduced the idea of mapping an isotropic Lagrangian pressure Hessian as upstream initial condition using the fluid deformation tensor. Recent work on a Gaussian fields closure, however, has shown that even Gaussian isotropic velocity fields contain significant anisotropy for the conditional pressure Hessian tensor due to the inherent velocity-pressure couplings, and that assuming an isotropic pressure Hessian as upstream condition may not be realistic. In this paper, Gaussian isotropic field statistics are used to generate more physical upstream conditions for the recent fluid deformation mapping. In this new framework, known isotropy relations can be satisfied a priori and no DNS-tuned coefficients are necessary. A detailed comparison of results from the new model, referred to as the recent deformation of Gaussian fields (RDGF) closure, with existing models and DNS shows the improvements gained, especially in various single-time statistics of the velocity gradient tensor at moderate Reynolds numbers. Application to arbitrarily high Reynolds numbers remains an open challenge for this type of model, however.
\end{abstract}

\section{Introduction}

The statistics of velocity gradients in isotropic turbulence are of both practical and theoretical importance in the study of turbulent flows (Sreenivasan \& Antonia 1997; Wallace 2009). The hypothesis of approximate local isotropy at sufficiently high Reynolds number (Kolmogorov 1941) provides an important framework for exploring universality of small-scale statistics, including velocity gradients, across a wide range of flows. It is well accepted that the dynamics of turbulence, including velocity gradients, can be better understood in a Lagrangian frame following the flow (Falkovich et al. 2001; Toschi \& Bodenschatz 2009). Also, in many practical situations, the velocity gradient along Lagrangian or inertial particle paths determines the dynamics of sub-Kolmogorov scale objects immersed in turbulent flows, such as the deformation and break-up of bubbles and immiscible drops (Biferale et al. 2010; Maniero et al. 2012; Biferale et al. 2014), the

$\dagger$ Email address for correspondence: pjohns86@jhu.edu 
stretching of polymers (Balkovsky et al. 2000; Chertkov 2000; Bagheri et al. 2012), the rotation rate and nutrient uptake of small swimming organisms (Batchelor 1980; Pedley \& Kessler 1992; Karp-Boss et al. 1996; Parsa et al. 2012; Chevillard \& Meneveau 2013), and hemolysis in red-blood cells (Arora et al. 2004; Behbahani et al. 2009; De Tullio et al. 2012), among other applications.

Meanwhile, from a theoretical perspective, the statistics of velocity gradients and increments in isotropic turbulence are key ingredients in exploring internal intermittency and multi-fractality (Kolmogorov 1962; Oboukhov 1962; Parisi \& Frisch 1985; Meneveau \& Sreenivasan 1991). In recent decades, the Lagrangian view of intermittency in turbulence has become a topic of increasing interest (Falkovich et al. 2001; Toschi \& Bodenschatz 2009). The energy cascade has been posed in the Lagrangian frame (Meneveau \& Lund 1994; Yu \& Meneveau 2010) and Lagrangian multi-fractality has been explored (Boffetta et al. 2002; Chevillard et al. 2003; Biferale et al. 2004, 2008; Arnèodo et al. 2008). While analysis methods for dynamical systems are often impractical because of the high-dimensionality of turbulent flows (Frisch 1995), tools such as finite-time Lyapunov exponents are useful in the Lagrangian frame for studying chaotic advection (Ottino 1989) and coherent structures (Haller 2000; Haller \& Yuan 2000; Green et al. 2007; Haller 2015).

The evolution of velocity gradients along Lagrangian paths contains two unclosed terms requiring models: the deviatoric part of the pressure Hessian and the viscous Laplacian. Removal of these two terms results in the restricted Euler equation, which has the unfortunate property of leading to a finite-time singularity (Vieillefosse 1982, 1984; Cantwell 1992). The term driving the singularity is the quadratic self-amplification of velocity gradients that is kinematic in nature. The unclosed terms are evidently responsible for opposing the restricted Euler singularity. A number of studies have shed some light on the dynamics of velocity gradients along Lagrangian paths, exploring invariant spaces and the unclosed terms (Nomura \& Post 1998; Martin et al. 1998a; Ooi et al. 1999; Lüthi et al. 2009; Lawson \& Dawson 2015).

Meanwhile, as reviewed in Meneveau (2011), attempts at closure models for the ODE governing the Lagrangian evolution of the velocity gradient tensor have enjoyed some success. The addition of a linear relaxation term eliminates the singularity for some initial conditions, but not for all (Martin et al. 1998b). Girimaji \& Pope (1990) introduced a model for the pressure Hessian and viscous Laplacian designed to reproduce lognormal statistics for the pseudo-dissipation by construction. Jeong \& Girimaji (2003) constructed a non-linear relaxation model for the viscous Laplacian using the trace of the inverse Cauchy-Green tensor, neglecting the deviatoric part of the pressure Hessian. Chevillard \& Meneveau (2006) and Chevillard et al. (2008) used the insight of Jeong and Girimaji's viscous Laplacian and the tetrad model of Chertkov et al. (1999) to introduce the Recent Fluid Deformation (RFD) approximation, providing closure for both the viscous Laplacian and the deviatoric part of the pressure Hessian. The underlying concept in the RFD model is that the conditional pressure Hessian can be approximated by considering an initially isotropic tensor subjected to fluid deformation for a short time using a constant velocity gradient. It was demonstrated that the RFD closure was able to prevent the singularity and the resulting system could thus reproduce many wellknown velocity gradient characteristics, including trends over a small range of moderate $R e_{\lambda}$ (Chevillard \& Meneveau 2006; Chevillard et al. 2008; Chevillard \& Meneveau 2011). Increasing the Reynolds number further, however, led to unphysical results, which were studied in some detail (Martins-Afonso \& Meneveau 2010). Meanwhile, Suman \& Girimaji $(2009,2011)$ have worked on a similar modeling approach for the Lagrangian velocity gradient evolution in compressible flows . 
Wilczek \& Meneveau (2014) took a different approach to closure, using a Gaussian fields (GF) assumption to compute directly the conditional averages of the deviatoric part of the pressure Hessian and viscous Laplacian in incompressible, isotropic turbulence. When using the resulting pressure Hessian from the GF model, however, Wilczek \& Meneveau (2014) found that the model was too weak to prevent the singularity. To make the model work, the Gaussian-derived coefficients were tuned empirically using stochastic estimation based on DNS data. The resulting Enhanced Gaussian Fields (EGF) closure with the fitted parameters provided good predictions of velocity gradient statistics, rivaling those of the RFD model.

The GF closure thus elucidated an important point, that even in an isotropic Gaussian velocity field, the conditional pressure Hessian tensor is not an isotropic tensor. In this paper, we propose that the initial conditions for a recent deformation closure are better represented by those of an isotropic Gaussian velocity field than by assuming an isotropic tensor as in the RFD closure. With this insight, we develop a new pressure Hessian and viscous Laplacian model based on a recent-deformation mapping closure for incompressible turbulence that assumes the initial condition of the mapping to be an isotropic Gaussian velocity field.

More detailed background on the RFD and GF/EGF closures is presented briefly in $\S 2$. Following that, the new model based on recent deformations of Gaussian fields is introduced and explained in $\S 3$. After a brief explanation in $\S 4$ of numerical methods for the stochastic ODEs and for the DNS data to which results are compared, an examination of results is given in $\S 5$. The results of the new model are compared alongside RFD and EGF results with DNS data, and afterward appropriate conclusions are drawn.

\section{Background}

In this section, the equations for the Lagrangian evolution of the velocity gradient tensor are briefly summarized. After that, the closure approach based on the FokkerPlanck equation for the velocity gradient is reviewed. Within this paradigm, the prior RFD and GF closure models are explained.

\subsection{Lagrangian Velocity Gradient Evolution}

In this paper, we consider incompressible, Newtonian fluids with arbitrary solenoidal forcing. The gradient of the incompressible Navier-Stokes equations gives the evolution equation for velocity gradient tensor, $A_{i j}=\frac{\partial u_{i}}{\partial x_{j}}$,

$$
\frac{d A_{i j}}{d t}=-A_{i k} A_{k j}-P_{i j}+\nu \nabla^{2} A_{i j}+F_{i j}
$$

where $\frac{d}{d t}=\frac{\partial}{\partial t}+u_{k} \frac{\partial}{\partial x_{k}}$ represents the material derivative, $P_{i j}=\frac{\partial^{2} p}{\partial x_{i} \partial x_{j}}$ is the symmetric pressure Hessian tensor, and $F_{i j}=\frac{\partial f_{i}}{\partial x_{j}}$ is the trace-free gradient of the forcing. The first term on the right-hand side is the non-linear self-amplification term, which leads to the restricted Euler dynamics and a finite-time singularity (Vieillefosse 1982, 1984; Cantwell 1992). While this self-amplification term is closed, the pressure Hessian and viscous Laplacian terms are unclosed, requiring information from neighboring Lagrangian trajectories.

The incompressibility constraint, i.e. that the velocity gradient tensor should be tracefree, can be incorporated by evaluating the trace of the velocity gradient evolution equation, which yields the pressure Poisson equation, $P_{k k}=2 Q$, where $Q \equiv-\frac{1}{2} A_{k \ell} A_{\ell k}$. Solving the pressure Poisson equation and twice taking the gradient leads to (Ohkitani 
\& Kishiba 1995),

$$
P_{i j}(\mathbf{x}, t)=\frac{2}{3} Q(\mathbf{x}, t) \delta_{i j}+\iiint_{P . V .} \frac{Q(\mathbf{x}+\mathbf{r})}{2 \pi r^{3}}\left(\delta_{i j}-3 \frac{r_{i} r_{j}}{r^{2}}\right) d \mathbf{r} .
$$

The isotropic part of the pressure Hessian is local and closed, while the deviatoric part of the pressure Hessian, $P_{i j}^{(d)}$, is non-local and depends on the structure of the surrounding flow. Decomposition into isotropic and deviatoric parts gives

$$
\frac{d A_{i j}}{d t}=-\left(A_{i k} A_{k j}+\frac{2}{3} Q \delta_{i j}\right)-P_{i j}^{(d)}+\nu \nabla^{2} A_{i j}+F_{i j} .
$$

This tensor equation represents 9 differential equations for the 9 components of the velocity gradient tensor, of which 8 are independent.

The velocity gradient tensor can be written as a sum of symmetric and anti-symmetric parts, $A_{i j}=S_{i j}+\Omega_{i j}$, where $S_{i j}=\frac{1}{2}\left(A_{i j}+A_{j i}\right)$ is the strain-rate tensor and $\Omega_{i j}=$ $\frac{1}{2}\left(A_{i j}-A_{j i}\right)$ is the rotation rate tensor, which can be related to the vorticity, $\omega_{i}=$ $-\epsilon_{i j k} \Omega_{j k}$. Using this decomposition on the Lagrangian evolution equation (Nomura \& Post 1998),

$$
\begin{aligned}
\frac{d S_{i j}}{d t}=-\left(S_{i k} S_{k j}-\frac{1}{3} S_{k \ell} S_{k \ell} \delta_{i j}\right)- & \left(\Omega_{i k} \Omega_{k j}-\frac{1}{3} \Omega_{i k} \Omega_{k j} \delta_{i j}\right) \\
& -P_{i j}^{(d)}+\nu \nabla^{2} S_{i j}+F_{i j}^{(s)}, \\
\frac{d \Omega_{i j}}{d t}=-\left(S_{i k} \Omega_{k j}+\Omega_{i k} S_{k j}\right) & +\nu \nabla^{2} \Omega_{i j}+F_{i j}^{(a)},
\end{aligned}
$$

where $F_{i j}^{(s)}=\frac{1}{2}\left(F_{i j}+F_{j i}\right)$ and $F_{i j}^{(a)}=\frac{1}{2}\left(F_{i j}-F_{j i}\right)$, are the symmetric and antisymmetric parts of the forcing, respectively. In this way, we can separately view the evolution of the vorticity and the strain-rate, although the strong coupling in the nonlinear term is evident.

\subsection{Stochastic Model}

In order to model the Lagrangian evolution of the velocity gradient, a stochastic representation is taken (Girimaji \& Pope 1990; Chevillard et al. 2008; Wilczek \& Meneveau 2014). The stochastic approach is built on the evolution equation for the single-time probability density function (PDF) for the velocity gradient tensor,

$$
\begin{aligned}
\frac{\partial f(\mathcal{A} ; t)}{\partial t}= & -\frac{\partial}{\partial \mathcal{A}_{i j}}\left(\left[-\left(\mathcal{A}_{i k} \mathcal{A}_{k j}+\frac{2}{3} \mathcal{Q} \delta_{i j}\right)-\left\langle P_{i j}^{(d)} \mid \mathcal{A}\right\rangle+\left\langle\nu \nabla^{2} A_{i j} \mid \mathcal{A}\right\rangle\right] f(\mathcal{A} ; t)\right) \\
& +\frac{1}{2} D_{i j k \ell} \frac{\partial^{2} f(\mathcal{A} ; t)}{\partial \mathcal{A}_{i j} \partial \mathcal{A}_{k \ell}} .
\end{aligned}
$$

This Fokker-Planck equation for the PDF evolution is constructed from (2.3) using stochastic forcing. The related Langevin equation is,

$$
d A_{i j}=\left[-\left(A_{i k} A_{k j}+\frac{2}{3} Q \delta_{i j}\right)-\left\langle P_{i j}^{(d)} \mid \mathbf{A}\right\rangle+\left\langle\nu \nabla^{2} A_{i j} \mid \mathbf{A}\right\rangle\right] d t+d F_{i j},
$$

which provides a model for the Lagrangian velocity gradient dynamics provided the two conditional averages and the stochastic noise term can be specified. The stochastic forcing term, $d F_{i j}=b_{i j k \ell} d W_{k l}$, is built on a tensorial Wiener process, $\left\langle d W_{i j}\right\rangle=0$, $\left\langle d W_{i j} d W_{k \ell}\right\rangle=\delta_{i k} \delta_{j \ell} d t$, with diffusion tensor $D_{i j k \ell}=b_{i j m n} b_{k \ell m n}$. 
Furthermore, this tensorial stochastic ODE can be decomposed into symmetric and anti-symmetric components, as in (2.4) and (2.5),

$$
\begin{array}{r}
d S_{i j}=\left[-\left(S_{i k} S_{k j}-\frac{1}{3} S_{k \ell} S_{k \ell} \delta_{i j}\right)-\left(\Omega_{i k} \Omega_{k j}-\frac{1}{3} \Omega_{i k} \Omega_{k j} \delta_{i j}\right)\right. \\
\left.-\left\langle P_{i j}^{(d)} \mid \mathbf{S}, \boldsymbol{\Omega}\right\rangle+\left\langle\nu \nabla^{2} S_{i j} \mid \mathbf{S}, \boldsymbol{\Omega}\right\rangle\right] d t+d F_{i j}^{(s)} \\
d \Omega_{i j}=\left[-\left(S_{i k} \Omega_{k j}+\Omega_{i k} S_{k j}\right)+\left\langle\nu \nabla^{2} \Omega_{i j} \mid \mathbf{S}, \boldsymbol{\Omega}\right\rangle\right] d t+d F_{i j}^{(a)}
\end{array}
$$

In this system, $\Omega_{i j}$ has three independent variables with the requirement to remain anti-symmetric and $S_{i j}$ has five independent variables with the requirement to remain symmetric and trace-free. The symmetric and anti-symmetric stochastic forcing terms, in this view, can be chosen independent of each other and obeying these constraints. The details of the stochastic forcing term are given in Appendix A.

\subsection{Recent Fluid Deformation Closure}

The central idea in the RFD closure approach Chevillard \& Meneveau (2006) is to introduce a coordinate mapping based on material volume deformation in the recent Lagrangian history. Defining a Lagrangian trajectory as a map, $\mathcal{T}_{t_{0}, t}: \mathbf{X} \in \mathbb{R}^{3} \mapsto \mathbf{x} \in \mathbb{R}^{3}$, from an initial condition $\mathbf{X}$ at time $t_{0}$ to a position $\mathbf{x}$ at a later time $t$, then the Lagrangian trajectory evolves according to $\frac{d x_{i}}{d t}=u_{i}(\mathbf{x}, t)$, with initial condition $x_{i}\left(t_{0}\right)=X_{i}$, where the velocity field, $\mathbf{u}(\mathbf{x}, t)$, is a solution to the incompressible Navier-Stokes equations with appropriate boundary and initial conditions.

The evolution of an infinitesimal fluid volume in the vicinity of $\mathbf{x}$ can be described by the deformation tensor, $D_{i j}=\partial x_{i} / \partial X_{j}$, which is the sensitivity of the trajectory to initial position and evolves as $\frac{d D_{i j}}{d t}=A_{i k} D_{k j}$ with initial condition $D_{i j}\left(\mathbf{X}, t_{0}\right)=\delta_{i j}$. The general solution to this equation involves the time-ordered exponential, but approximating that the velocity gradient is constant for short time,

$$
\mathbf{D}\left(\mathbf{x}, t ; \mathbf{X}, t_{0}\right) \approx \exp \left[\mathbf{A}(\mathbf{x}, t)\left(t-t_{0}\right)\right]
$$

Instead of directly attempting to close the conditional averages in (2.7), first the approximate fluid deformation tensor is used to strain the coordinate system,

$$
\left\langle P_{i j} \mid \mathbf{A}\right\rangle=\left\langle\frac{\partial^{2} p}{\partial x_{i} \partial x_{j}} \mid \mathbf{A}\right\rangle \approx \frac{\partial X_{k}}{\partial x_{i}}\left\langle\frac{\partial^{2} p}{\partial X_{k} \partial X_{\ell}} \mid \mathbf{A}\right\rangle \frac{\partial X_{\ell}}{\partial x_{j}}=D_{k i}^{-1}\left\langle\widetilde{P}_{k \ell} \mid \mathbf{A}\right\rangle D_{\ell j}^{-1},
$$

where $\widetilde{P}_{i j}$ represents an approximation for the pressure Hessian at a previous time along the Lagrangian path and $D_{i j}^{-1}=\partial X_{i} / \partial x_{j} \approx\left(\exp \left[-\mathbf{A}(\mathbf{x}, t)\left(t-t_{0}\right)\right]\right)_{i j}$. This is akin to assuming the pressure approximately constant along pathlines for a short time (in the sense of conditional averages on $\mathbf{A}$ ), so that the changes in the conditional pressure Hessian are due entirely to the relative movement of neighboring fluid trajectories induced by the local velocity gradient. In this way, the closure of the conditional pressure Hessian requires the specification of initial conditions of the pressure Hessian upstream along the Lagrangian path.

The strongest assumption in the RFD model comes next, in assuming the initial condition for the mapping, i.e. the upstream conditional pressure Hessian, to be an isotropic tensor,

$$
\left\langle\widetilde{P}_{i j} \mid \mathbf{A}\right\rangle \approx \frac{1}{3}\left\langle\widetilde{P}_{k k} \mid \mathbf{A}\right\rangle \delta_{i j}
$$


which gives

$$
\left\langle P_{i j} \mid \mathbf{A}\right\rangle \approx \frac{1}{3} C_{i j}^{-1}\left\langle\widetilde{P}_{k k} \mid \mathbf{A}\right\rangle .
$$

where $C_{i j}^{-1}=D_{k i}^{-1} D_{k j}^{-1}$ is the inverse of the left Cauchy-Green tensor. The trace of (2.13),

$$
2 Q=\left\langle P_{k k} \mid \mathbf{A}\right\rangle \approx \frac{1}{3} C_{k k}^{-1}\left\langle\widetilde{P}_{\ell \ell} \mid \mathbf{A}\right\rangle
$$

upon substitution, allows for the final form,

$$
\left\langle P_{i j} \mid \mathbf{A}\right\rangle \approx 2 Q \frac{C_{i j}^{-1}}{C_{k k}^{-1}}
$$

This form of the conditional pressure Hessian is appealing due to its simplicity and the intuition that the statistical bias of the pressure Hessian is related to the recent deformation of fluid particles by the velocity gradient tensor. However, as will be recalled later, even isotropic Gaussian velocity fields contain anisotropic contributions for the conditional pressure Hessian, casting some doubts onto (2.12) above.

The RFD model treats the viscous Laplacian in the same way,

$$
\left\langle\nu \nabla^{2} A_{i j} \mid \mathbf{A}\right\rangle \approx \nu \frac{\partial X_{p}}{\partial x_{k}}\left\langle\frac{\partial A_{i j}}{\partial X_{p} \partial X_{q}} \mid \mathbf{A}\right\rangle \frac{\partial X_{q}}{\partial x_{k}}=\nu D_{p k}^{-1}\left\langle\frac{\partial A_{i j}}{\partial X_{p} \partial X_{q}} \mid \mathbf{A}\right\rangle D_{q k}^{-1},
$$

and assumes that the conditional Hessian of the velocity gradient tensor is likewise an isotropic tensor,

$$
\left\langle\frac{\partial A_{i j}}{\partial X_{k} \partial X_{\ell}} \mid \mathbf{A}\right\rangle=\frac{1}{3}\left\langle\nabla_{\mathbf{X}}^{2} A_{i j} \mid \mathbf{A}\right\rangle \delta_{k \ell},
$$

which yields,

$$
\left\langle\nu \nabla^{2} A_{i j} \mid \mathbf{A}\right\rangle \approx \frac{C_{k k}^{-1}}{3} \nu\left\langle\nabla_{\mathbf{X}}^{2} A_{i j} \mid \mathbf{A}\right\rangle .
$$

Then taking a linear relaxation model (Martin et al. 1998b) for the initial upstream conditions of the conditional viscous Laplacian,

$$
\left\langle\nu \nabla^{2} A_{i j} \mid \mathbf{A}\right\rangle \approx-\frac{C_{k k}^{-1}}{3} \frac{A_{i j}}{T} .
$$

In this way, the recent deformation provides a physically motivated mechanistic approach to introduce non-linearity in the viscous Laplacian term, which helpful in removing the finite-time singularity (the Cauchy-Green tensor is exponential rather than linear). Chevillard \& Meneveau (2006) and Chevillard et al. (2008) argue that the proper relaxation timescale, $T$, for the viscous Laplacian is the integral timescale, and that the proper timescale for the recent deformation is the Kolmogorov timescale, $t-t_{0}=\tau_{\eta}$. In this way, the model introduces $R e_{\lambda} \sim\left(T / \tau_{\eta}\right)$ effects. Indeed, certain intermittency trends are reproduced by this model (Chevillard \& Meneveau 2006) at moderate $R e_{\lambda}$, but continuing to increase $R e_{\lambda}$ beyond a certain threshold leads to increasingly unphysical results (Martins-Afonso \& Meneveau 2010). Nonetheless, the RFD closure provides a model that reproduced many of the known trends of velocity gradient statistics at moderate $R e_{\lambda}$, and continues to be useful for studying velocity gradient statistics (Moriconi et al. 2014).

\subsection{Gaussian Fields Closure}

Wilczek \& Meneveau (2014) took a different approach to closing the conditional averages. They assumed that the velocity field has joint-Gaussian N-point PDFs with 
prescribed spectral (two-point) statistics (the pressure field constructed from such a velocity field is not necessarily Gaussian). They computed the conditional averages using this approximation by employing the characteristic functional of a Gaussian velocity field and obtaining an exact analytical result for the conditional pressure Hessian for a Gaussian velocity field

$$
\begin{aligned}
\left\langle P_{i j}^{(d)} \mid \mathbf{A}\right\rangle_{\text {Gaussian }}= & \alpha\left(S_{i k} S_{k j}-\frac{1}{3} S_{k \ell} S_{\ell k} \delta_{i j}\right)+\beta\left(\Omega_{i k} \Omega_{k j}-\frac{1}{3} \Omega_{k \ell} \Omega_{\ell k} \delta_{i j}\right) \\
& +\gamma\left(S_{i k} \Omega_{k j}-\Omega_{i k} S_{k j}\right)
\end{aligned}
$$

where

$$
\alpha=-\frac{2}{7}, \quad \beta=-\frac{2}{5}, \quad \gamma=\frac{6}{25}+\frac{16}{75 f^{\prime \prime}(0)^{2}} \int \frac{f^{\prime}(r) f^{\prime \prime \prime}(r)}{r} d r,
$$

with $f(r)$ specifying the longitudinal velocity correlation function in isotropic turbulence. In this expression, $\alpha$ and $\beta$ are independent of $R e_{\lambda}$ while $\gamma$ is expected to have weak $R e_{\lambda}$-dependence through the integral of the correlation function derivatives. Using a model spectrum at $R e_{\lambda}=430$, a numerical result of $\gamma \approx 0.08$ was obtained (Wilczek \& Meneveau 2014) .

Furthermore, the conditional viscous Laplacian could also be computed for Gaussian fields (Wilczek \& Meneveau 2014),

$$
\left\langle\nu \nabla^{2} A_{i j} \mid \mathbf{A}\right\rangle_{\text {Gaussian }}=\delta A_{i j}, \quad \text { where } \quad \delta=\nu \frac{7}{3} \frac{f^{(4)}(0)}{f^{\prime \prime}(0)} .
$$

Note that $\delta<0$ for realistic correlation functions, meaning that the Gaussian approximation leads to a linear damping model as in Martin et al. (1998b) for the viscous Laplacian. Numerical evaluation using a model spectrum at $R e_{\lambda}=430$ gave the result $\delta \approx-0.65 / \tau_{\eta}$. Using the above Gaussian-derived functional form but invoking in addition the balance of enstrophy production and dissipation with its relationship to skewness, Wilczek \& Meneveau (2014) related the coefficient $\gamma$ to the velocity derivative skewness, $\mathcal{S}=\left\langle\left(\partial u_{1} / \partial x_{1}\right)^{3}\right\rangle /\left\langle\left(\partial u_{1} / \partial x_{1}\right)^{2}\right\rangle^{3 / 2}$,

$$
\delta=\frac{7}{6 \sqrt{15}} \frac{\mathcal{S}}{\tau_{\eta}}
$$

a result which gave much better agreement values estimated from DNS at $R e_{\lambda}=430$, namely $\delta \approx-0.15 / \tau_{\eta}$, when using realistic values for the skewness (non-zero, i.e. nonGaussian). Because the original Gaussian closure led to a singularity when integrated numerically, the authors considered an alternative model in which the functional form of the Gaussian closure was retained by the coefficients were empirically obtained by estimating them from DNS results: $\alpha=-0.61, \beta=-0.65, \gamma=0.14, \delta=-0.15 / \tau_{\eta}$. With these empirically-adjusted coefficients, statistical stationarity was achieved and many of the known trends for velocity gradient statistics were reproduced with this approach termed the Enhanced Gaussian Fields (EGF) closure.

\section{Recent Deformation of Gaussian Fields Mapping Closure}

This section introduces the RDGF closure for the pressure Hessian and viscous Laplacian terms in the Lagrangian stochastic evolution equation for the velocity gradient tensor. 


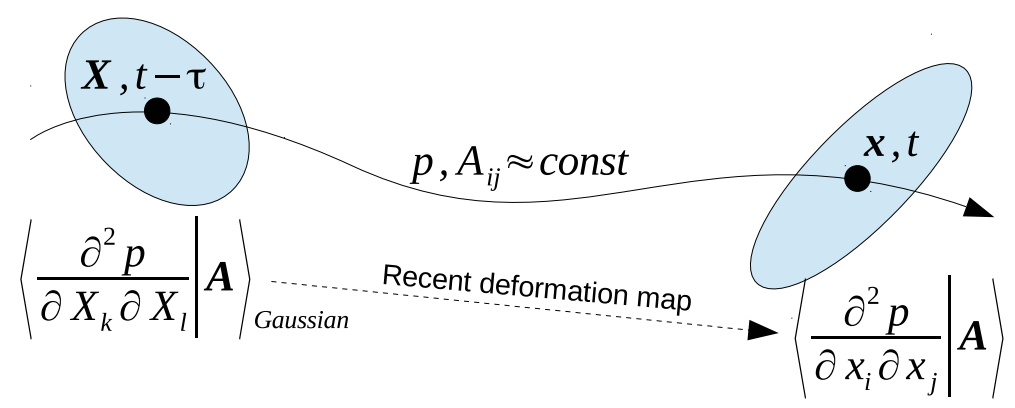

FIGURE 1. Schematic illustrating the main elements of the RDGF model for the conditional pressure Hessian. The viscous Laplacian model is constructed analogously.

\subsection{Overview}

As summarized before, a strong assumption underlying the RFD approximation was the assumption that the initial upstream condition of the conditional pressure Hessian (and viscous Laplacian) are isotropic tensors. Here we relax this strong assumption and instead assume that the upstream conditional pressure Hessian is that of an isotropic Gaussian velocity field. In this way, (2.12) is modified as follows

$$
\left\langle\widetilde{P}_{i j} \mid \mathbf{A}\right\rangle \approx \frac{1}{3}\left\langle\widetilde{P}_{k k} \mid \mathbf{A}\right\rangle \delta_{i j}+\left\langle\widetilde{P}_{i j}^{(d)} \mid \mathbf{A}\right\rangle_{\text {Gaussian }},
$$

where the latter term is evaluated using (2.20). Similarly for the viscous term, the conditional Hessian of the upstream velocity gradient is no longer assumed isotropic, and (2.17) is modified to include the anisotropic contributions from the Gaussian closure. The same mapping as in the RFD model is applied to convert the upstream initial conditions to the resulting closure. Figure 1 illustrates the overall procedure for constructing the model for the pressure Hessian. A similar procedure is used for the viscous Laplacian.

We name this approach the Recent Deformation of Gaussian Fields (RDGF) model. In the sense of this nomenclature, the term 'Gaussian fields' is used to refer to the Gaussian velocity field along with its associated (non-Gaussian) pressure field. For the pressure Hessian, the recent deformation mapping is applied to the pressure field derived from Gaussian velocity field.

The underlying phenomenology of the RDGF model is that approximate turbulence statistics can be developed efficiently by a mapping of Gaussian statistics. This motivation is similar to the ideas behind the mapping closures (Chen et al. 1989; Kraichnan 1990; Pope 1991), as well as the multiscale turnover Lagrangian map (MTLM) procedure of Rosales \& Meneveau (2008) to generate non-Gaussian synthetic turbulence fields.

\subsection{Model Details}

The model for the unclosed terms along the Lagrangian path at point $\mathbf{x}$ (time $t$ ) involves applying the Gaussian fields approximation at the upstream point $\mathbf{X}$ (time $t-\tau$ ). For the deviatoric part of the pressure Hessian, using (2.20),

$$
\begin{aligned}
\left\langle\widetilde{P}_{i j}^{(d)} \mid \mathbf{A}\right\rangle_{\text {Gaussian }}= & \alpha\left(S_{i k} S_{k j}-\frac{1}{3} S_{k \ell} S_{\ell k} \delta_{i j}\right)+\beta\left(\Omega_{i k} \Omega_{k j}-\frac{1}{3} \Omega_{k \ell} \Omega_{\ell k} \delta_{i j}\right) \\
& +\gamma\left(S_{i k} \Omega_{k j}-\Omega_{i k} S_{k j}\right)
\end{aligned}
$$

where (2.21) provides the numerical values of the parameters for Gaussian fields. In Appendix B, an analytical evaluation of $\gamma$ using Batchelor interpolation for the second- 
order structure function is presented (Batchelor 1951). The result, $\gamma=\frac{86}{1365} \approx 0.063$, does not deviate much from the previous numerical result (Wilczek \& Meneveau 2014).

Similarly, the Gaussian fields approximation for the upstream Hessian of the velocity gradient uses the results of Appendix C,

$$
\begin{aligned}
\left\langle\nu \frac{\partial^{2} A_{i j}}{\partial X_{p} \partial X_{q}} \mid \mathbf{A}\right\rangle_{\text {Gaussian }}= & \delta\left[T_{i j} \delta_{p q}+T_{i q} \delta_{j p}+T_{i p} \delta_{j q}\right. \\
& \left.-\frac{2}{21}\left(S_{j q} \delta_{i p}+S_{j p} \delta_{i q}+S_{p q} \delta_{i j}\right)\right]
\end{aligned}
$$

where

$$
\delta=\nu \frac{7}{3} \frac{f^{(4)}(0)}{f^{\prime \prime}(0)}, \quad T_{i j}=\frac{23}{105} A_{i j}+\frac{2}{105} A_{j i}, \quad S_{i j}=\frac{1}{2}\left(A_{i j}+A_{j i}\right) .
$$

It can be easily shown that contraction with $\delta_{p q}$ recovers (2.22) and contraction with $\delta_{i j}, \delta_{i p}$, or $\delta_{i q}$ causes the term to vanish in accordance with incompressibility. Following Wilczek \& Meneveau (2014), i.e. (2.23), the enstrophy balance is used to determine $\delta$ in Appendix D,

$$
\delta=\frac{C_{k k}}{3} \frac{7}{6 \sqrt{15}} \frac{\mathcal{S}}{\tau_{\eta}},
$$

where the typical value of $\mathcal{S}=-0.6$ can be used.

Then, the conditional pressure Hessian and velocity gradient Hessian are mapped from $\mathbf{X}$ to $\mathbf{x}$ along the trajectory. The deformation tensor used for the mapping, $D_{i j}=\frac{\partial x_{i}}{\partial X_{j}}$, is approximated by assuming that the velocity gradient is constant for the short time span $\tau$, i.e. (2.10). Using (2.11) with the new upstream conditional pressure Hessian in (3.1),

$$
\left\langle P_{i j} \mid \mathbf{A}\right\rangle=\frac{1}{3}\left\langle\widetilde{P}_{\ell \ell} \mid \mathbf{A}\right\rangle C_{i j}^{-1}+D_{m i}^{-1}\left\langle\widetilde{P}_{m n}^{(d)} \mid \mathbf{A}\right\rangle_{\text {Gaussian }} D_{n j}^{-1},
$$

where (3.2) is substituted for the deviatoric part of the pressure Hesssian. The trace of this equation gives,

$$
2 Q=\left\langle P_{k k} \mid \mathbf{A}\right\rangle=D_{m k}^{-1}\left\langle\widetilde{P}_{m n}^{(d)} \mid \mathbf{A}\right\rangle D_{n k}^{-1}+\frac{1}{3}\left\langle\widetilde{P}_{\ell \ell} \mid \mathbf{A}\right\rangle C_{k k}^{-1}
$$

Solving (3.7) for $\left\langle\widetilde{P}_{k k} \mid \mathbf{A}\right\rangle$, and substituting into (3.6), the resulting closure is,

$$
\left\langle P_{i j} \mid \mathbf{A}\right\rangle=2 Q \frac{C_{i j}^{-1}}{C_{k k}^{-1}}+G_{i j}-\frac{C_{i j}^{-1}}{C_{k k}^{-1}} G_{\ell \ell}
$$

where

$$
G_{i j}=D_{m i}^{-1}\left\langle\widetilde{P}_{m n}^{(d)} \mid \mathbf{A}\right\rangle_{\text {Gaussian }} D_{n j}^{-1},
$$

using (3.2) with (2.21). Similarly for the viscous Laplacian, using (2.16) with the new upstream conditional viscous Hessian (3.3) leads to,

$$
\left\langle\nu \nabla^{2} A_{i j} \mid \mathbf{A}\right\rangle=\delta\left(T_{i j} C_{k k}^{-1}+2 T_{i k} B_{k j}^{-1}-\frac{4}{21} B_{i k}^{-1} S_{k j}-\frac{2}{21} B_{k \ell}^{-1} S_{k \ell} \delta_{i j}\right)
$$

where $B_{i j}^{-1}=D_{i k}^{-1} D_{j k}^{-1}$ is the inverse of the right Cauchy-Green tensor and $\mathbf{T}$ and $\mathbf{S}$ are given in (3.4). 


\subsection{The Resulting Model}

The resulting stochastic ODE model for the Lagrangian velocity gradient dynamics is

$$
d A_{i j}=\left[-\left(A_{i k} A_{k j}-\frac{C_{i j}^{-1}}{C_{k k}^{-1}} \operatorname{tr}\left(\mathbf{A}^{2}\right)\right)-\left(G_{i j}-\frac{C_{i j}^{-1}}{C_{k k}^{-1}} \operatorname{tr}(\mathbf{G})\right)+V_{i j}\right] d t+b_{i j k \ell} d W_{i j},
$$

where the contribution of the deviatoric part of the back-in-time pressure Hessian is,

$$
\begin{aligned}
G_{i j}= & D_{m i}^{-1}\left[-\frac{2}{7}\left(S_{m k} S_{k n}-\frac{1}{3} S_{k \ell} S_{\ell k} \delta_{m n}\right)-\frac{2}{5}\left(\Omega_{m k} \Omega_{k n}-\frac{1}{3} \Omega_{k \ell} \Omega_{\ell k} \delta_{m n}\right)\right. \\
& \left.+\frac{86}{1365}\left(S_{m k} \Omega_{k n}-\Omega_{m k} S_{k n}\right)\right] D_{n j}^{-1}
\end{aligned}
$$

and the contribution of the viscous Laplacian is,

$$
V_{i j}=\frac{7}{6 \sqrt{15}} \frac{C_{k k}}{3} \frac{\mathcal{S}}{\tau_{\eta}}\left(T_{i j} C_{k k}^{-1}+2 T_{i k} B_{k j}^{-1}-\frac{4}{21} B_{i k}^{-1} S_{k j}-\frac{2}{21} B_{k \ell}^{-1} S_{k \ell} \delta_{i j}\right),
$$

with $\mathcal{S}=-0.6$ and,

$$
S_{i j}=\frac{1}{2}\left(A_{i j}+A_{j i}\right), \quad \Omega_{i j}=\frac{1}{2}\left(A_{i j}-A_{j i}\right), \quad T_{i j}=\frac{23}{105} A_{i j}+\frac{2}{105} A_{j i} .
$$

The recent deformation is described by

$$
D_{i j}^{-1}=[\exp (-\mathbf{A} \tau)]_{i j}, \quad C_{i j}^{-1}=D_{k i}^{-1} D_{k j}^{-1}, \quad B_{i j}^{-1}=D_{i k}^{-1} D_{j k}^{-1},
$$

and the diffusion coefficient tensor of the stochastic forcing term is

$$
b_{i j k \ell}=-\frac{1}{3} \sqrt{\frac{D_{s}}{5}} \delta_{i j} \delta_{k \ell}+\frac{1}{2}\left(\sqrt{\frac{D_{s}}{5}}+\sqrt{\frac{D_{a}}{3}}\right) \delta_{i k} \delta_{j \ell}+\frac{1}{2}\left(\sqrt{\frac{D_{s}}{5}}-\sqrt{\frac{D_{a}}{3}}\right) \delta_{i \ell} \delta_{j k} .
$$

Note that the present model does not use the coefficients estimated from DNS. Instead, the coefficients are used as derived from the Gaussian field statistics.

In some sense, this model can be seen as a generalization of both RFD and GF closures. To recover the RFD model, first the back-in-time deviatoric component of the pressure Hessian should be removed, $G_{i j}=0$, i.e. $\alpha=\beta=\gamma=0$. Then, including only the isotropic part of (3.3) gives $\nu \nabla^{2} A_{i j}=\delta \frac{C_{k k}^{-1}}{3} A_{i j}$, and the coefficient should be set to $\delta=-\frac{1}{T}$, where $T$ is the integral timescale. This roughly corresponds to the RFD model at $\frac{\tau_{K}}{T}=-\frac{7 \mathcal{S}}{6 \sqrt{15}} \approx 0.18$. To recover the GF model, the deformation tensor should be set to identity, $D_{i j}=\delta_{i j}$.

\subsection{Parameters and Constraints}

The model now contains three parameters that have yet to be determined: $D_{s}$, $D_{a}$, and $\tau$. As discussed in more detail in Appendix A, the stochastic forcing term, $d F_{i j}=b_{i j k \ell} d W_{k \ell}$, can be split into symmetric and anti-symmetric parts, each with its own amplitude. This can be thought of as separately forcing Eqs (2.8) and (2.9). The amplitudes of the symmetric and anti-symmetric stochastic forcing tensors, $D_{s}$ and $D_{a}$, are two parameters that must be set to fully specify the model.

Additionally, the time interval of the mapping, $\tau$, must be set. In keeping with the RFD phenomenology, it is expected that this should be $\tau \sim \mathcal{O}\left(\tau_{\eta}\right)$. The RFD model used $\tau=\tau_{K}$, where $\tau_{K}$ is an input Kolmogorov timescale, but a posteriori evaluation at $\frac{\tau_{K}}{T}=0.1$ reveals that the actual Kolmogorov timescale produced by the model is 
$\tau_{\eta} \approx 2.0 \tau_{K}$. Therefore, the effective time interval was $\tau \approx 0.5 \tau_{\eta}$, based on the actual velocity gradient statistics produced by the model.

The three free parameters can be set by a choice of three constraints. First, without loss of generality, considering the evolution of the dimensionless velocity gradient tensor, $\left\langle S_{i j} S_{i j}\right\rangle=\frac{1}{2}$. This constraint effectively guarantees that the definition of $\delta$ in terms of $\tau_{\eta}$ is consistent. For the other two constraints, it is desirable to pick relationships for isotropic turbulence with analytical derivation, which can be considered a priori constraints. It is natural, then, to pick the two Betchov relations (Betchov 1956), $\langle Q\rangle=\langle R\rangle=0$. In light of the aforementioned dimensionless form of the equation, the former can be rephrased as $\left\langle\Omega_{i j} \Omega_{i j}\right\rangle=\frac{1}{2}$.

The determination of the three parameters using the three constraints can be posed as a three-dimensional root-finding problem. The appropriate values for the parameters were found empirically by numerical solutions of the model (see $\S 4.1$ below for details) using Broyden's method (Press et al. 1992). The procedure involved iteratively adjusting $D_{s}, D_{a}$, and $\tau$ and evaluating sufficiently converged statistics of $\left\langle S_{i j} S_{i j}\right\rangle,\left\langle\Omega_{i j} \Omega_{i j}\right\rangle$ and $\langle R\rangle$ from the numerical solutions of the model until the constraints were satisfied with the desired accuracy (four decimal places). The iterative method for determining the correct model parameters converges toward,

$$
D_{s}=0.1014 / \tau_{\eta}^{3}, \quad D_{a}=0.0505 / \tau_{\eta}^{3}, \quad \tau=0.1302 \tau_{\eta} .
$$

The mapping time is considerably shorter than that of RFD closure because the additional deviatoric part of the pressure Hessian was added to the RFD model, which was by itself already strong enough to counter the singularity with $\tau \approx 0.5 \tau_{\eta}$.

\section{Numerical Methods}

\subsection{Stochastic Differential Equation Solver}

The three models introduced in the previous sections (RFD, EGF and RDGF) can be advanced numerically as a system of stochastic ODEs. A second-order predictorcorrector method is used for time advancement. Time steps of size $d t / \tau_{\eta}=0.04,0.02$, and 0.01 are compared to verify discretization convergence. Ensembles of $2^{16}$ trajectories are advanced for $1000 \tau_{\eta}$ to achieve convergence of desired statistical quantities (up to fourth-order moments). Without loss of generality, $\tau_{\eta}=1$ was used for all runs. The Fortran simulations are performed in serial and run on a desktop machine, taking a few hours to complete.

\subsection{Direct Numerical Simulation Database}

The Johns Hopkins Turbulence Databases (JHTDB) isotropic dataset (Li et al. 2008; Perlman et al. 2007) provided the DNS statistics used for most of the comparisons in this paper. The dataset contains the simulation results from a $R e_{\lambda}=430$ simulation of Navier-Stokes with forcing at the two lowest wavenumbers. The pseudo-spectral simulation provided a $1024^{3}$ resolution on a $(2 \pi)^{3}$ cubic domain. Time advancement was accomplished via the $2^{\text {nd }}$-order Adams-Bashforth scheme and de-aliasing was done with $2 \sqrt{2} / 3$ truncation and random phase shift. In a few cases, the comparisons are supplemented with another DNS at $R e_{\lambda}=160$ using the same simulation code. Important parameters for the simulations are given in Table 1. It is worth noting that RFD model with $\frac{\tau_{K}}{T}=0.1$ has been equated with $R e_{\lambda}=150$ (Chevillard et al. 2008). Reaching $R e_{\lambda}=430$ requires $\frac{\tau_{K}}{T} \approx 0.035$, which is outside the range for which RFD model produces results with reasonable accuracy. Therefore, in this paper, we use $\frac{\tau_{K}}{T}=0.1$ for the RFD simulations, the value at which the RFD model seems to perform the best. 


\begin{tabular}{cccccccc}
$\mathrm{N}$ & $R e_{\lambda}$ & $\epsilon$ & $\nu$ & $\eta$ & $\tau_{\eta}$ & $\Delta t$ & $k_{\max } \eta$ \\
\hline $256^{3}$ & 160 & 0.112 & $1.2 \mathrm{e}-03$ & $1.11 \mathrm{e}-02$ & 0.104 & $5 \mathrm{e}-04$ & 1.34 \\
$1024^{3}$ & 430 & 0.093 & $1.85 \mathrm{e}-04$ & $2.87 \mathrm{e}-03$ & 0.045 & $2 \mathrm{e}-04$ & 1.39
\end{tabular}

TABLE 1. Numerical details for simulations used in this paper.
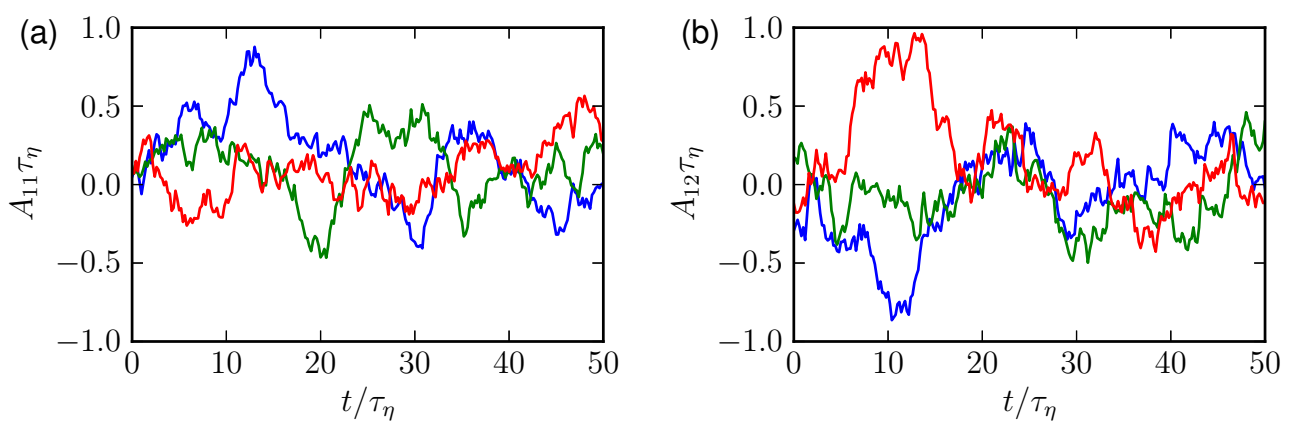

Figure 2. Sample trajectories of (a) longitudinal and (b) transverse velocity gradient components from the RDGF mapping closure.

\section{Results}

\subsection{Longitudinal and Transverse Components}

Figure 2 illustrates the output of the RDGF mapping closure by plotting sample trajectories of longitudinal and transverse velocity components over an interval of $20 \tau_{\eta}$. Because of the stochastic forcing, the paths appear rough, even at the scale of the Kolmogorov timescale. Nonetheless, such stochastic models can be useful when their statistical behavior provides a good model for Lagrangian velocity gradient statistics in isotropic turbulence.

The probability density functions for the longitudinal velocity derivative, $A_{11}$, and transverse velocity derivative, $A_{12}$, are shown in figure 3 . The RFD, EGF, and RDGF closures are compared with DNS results at the two different Reynolds numbers. The negative skewness expected for $A_{11}$ and the symmetry expected for $A_{12}$ are reflected by all three models. The RFD results appear much too close to Gaussian when compared with DNS results. The longitudinal velocity gradient distributions (top row of figure) from the EGF and RDGF models are better than that of RFD in terms of deviation from Gaussian behavior. For the transverse component, the RFD and EGF results appear similar, being between Gaussian and the DNS results. The RDGF mapping closure provides a much better match for the $A_{12}$ PDF. The trends suggest that the RDGF model may provide an even better fit for DNS data at slightly lower $R e_{\lambda}$, but we refrain from any iterative matching with any particular precise value of $R e_{\lambda}$ as we are mostly interested in overall trends.

As a compact comparison, Table 2 records the skewness and flatness factors of the above PDFs. All three models significantly under-predict the magnitude of the negative skewness for $A_{11}$, though the RFD and RDGF mapping closures are much closer than the EGF closure. The flatness factors for the longitudinal and transverse components help quantify the tendency of the model to reproduce the fattened tails of the PDFs in figure 3. For the longitudinal component, the EGF model appears to give the closest match, 

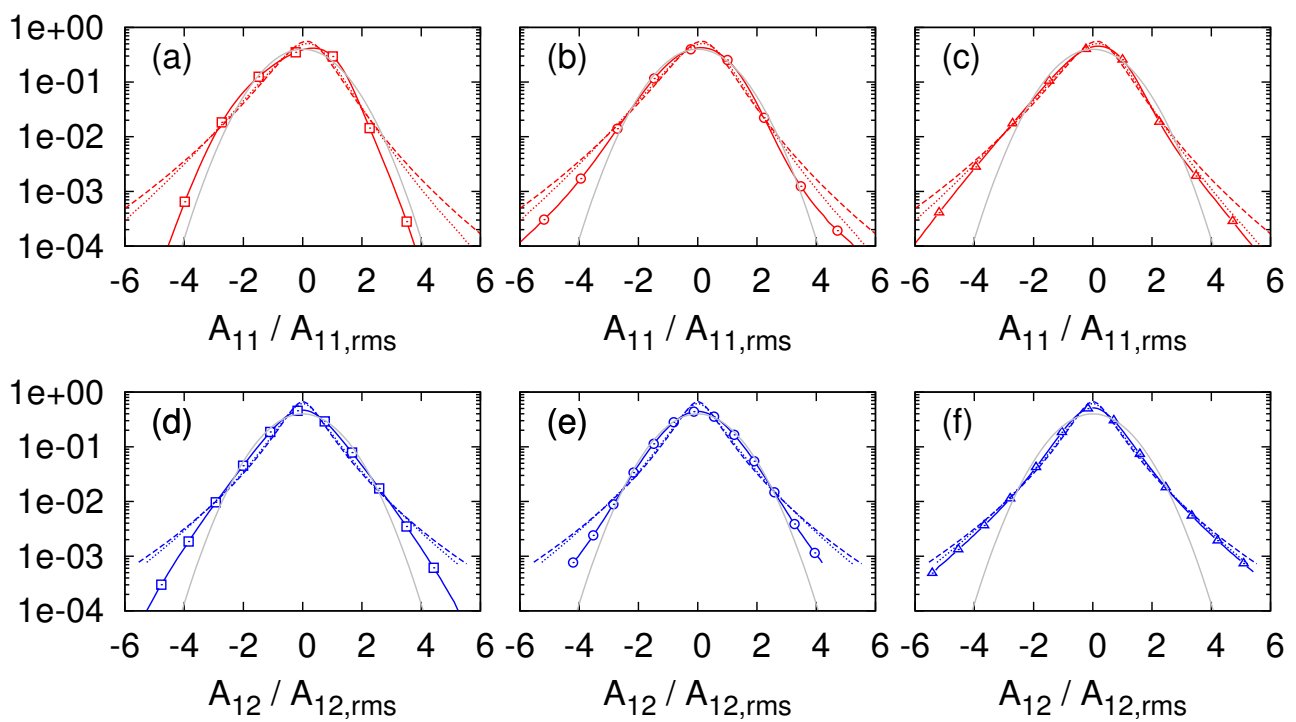

Figure 3. Single component PDFs for longitudinal (a-c) and transverse (d-f) velocity components. Three models are compared: (a,d) RFD, (b,e) EGF, (c,f) RDGF mapping closure. Solid gray line denotes Gaussian, dashed line shows DNS results at $R e_{\lambda}=430$, dotted line shows DNS at $R e_{\lambda}=160$, and solid line with markers shows the model result.

\begin{tabular}{ccccc} 
& $\frac{\left\langle A_{11}^{3}\right\rangle}{\left\langle A_{11}^{2}\right\rangle^{3 / 2}}$ & $\frac{\left\langle A_{12}^{3}\right\rangle}{\left\langle A_{12}^{2}\right\rangle^{3 / 2}}$ & $\frac{\left\langle A_{11}^{4}\right\rangle}{\left\langle A_{11}^{2}\right\rangle^{2}}$ & $\frac{\left\langle A_{12}^{4}\right\rangle}{\left\langle A_{12}^{2}\right\rangle^{2}}$ \\
\hline RFD & -0.44 & 0.0 & 3.2 & 4.3 \\
EGF & -0.31 & 0.0 & 6.5 & 6.3 \\
RDGF & -0.45 & 0.0 & 4.7 & 6.8 \\
$R e_{\lambda}=160$ & -0.52 & 0.0 & 5.9 & 9.4 \\
$R e_{\lambda}=430$ & -0.60 & 0.0 & 8.5 & 13.2
\end{tabular}

TABLE 2. Skewness and kurtosis values for longitudinal and transverse velocity gradient components from each model compared with DNS.

while RDGF is slightly closer for the transverse component. In each case, the flatness factors are too low, as was probably already evident in the above figures. It appears that the trend in the RFD and RDGF mapping closures that the longitudinal component has lower flatness than the transverse component better reflects the DNS trend. Indeed, as was discussed above, these results for RDGF could be seen as somewhat more appropriate for matching the DNS results at even lower Reynolds number.

\subsection{Isotropic Relations}

Table 3 compares the extent to which each of the models is able to reproduce important isotropy relations. Each ratio is equal to unity for isotropic turbulence. The first ratio, $\frac{\left\langle S_{i j} S_{i j}\right\rangle}{\left\langle\Omega_{i j} \Omega_{i j}\right\rangle}$, represents the ratio of strain-rate magnitude to vorticity magnitude produced by the model and is equal to unity since by construction (adjustment of forcing parameters), $\langle Q\rangle=0$. The second ratio, $\frac{-\frac{1}{3}\left\langle S_{i j} S_{j k} S_{k i}\right\rangle}{\frac{1}{4}\left\langle\omega_{i} S_{i j} \omega_{j}\right\rangle}$, represents the balance between strain production and vorticity production and is equal to unity if $\langle R\rangle=0$, also expected 


\begin{tabular}{ccccc} 
& $\frac{\left\langle S_{i j} S_{i j}\right\rangle}{\left\langle\Omega_{i j} \Omega_{i j}\right\rangle}$ & $\frac{-\frac{1}{3}\left\langle S_{i j} S_{j k} S_{k i}\right\rangle}{\frac{1}{4}\left\langle\omega_{i} S_{i j} \omega_{j}\right\rangle}$ & $\frac{15\left\langle A_{11}^{2}\right\rangle}{2\left\langle S_{i j} S_{i j}\right\rangle}$ & $\frac{-\frac{35}{2}\left\langle A_{11}^{3}\right\rangle}{\left\langle\omega_{i} S_{i j} \omega_{j}\right\rangle}$ \\
\hline RFD & 1.143 & 1.76 & 1.00 & 1.76 \\
EGF & 0.486 & 0.52 & 1.00 & 0.46 \\
RDGF & 1.000 & 1.00 & 1.00 & 1.00
\end{tabular}

TABLE 3. Results for competing models in terms of reproducing known isotropic relations.

due to the adjustment of forcing parameters. The identities are all satisfied within numerical error showing that the numerical tuning of the three parameters $\left(D_{s}, D_{a}\right.$, and $\tau$ ) is very accurate. This represents a significant advantage of the RDGF mapping closure, seeing that the earlier RFD model slightly over-emphasizes strain-dominant and strain-production-dominant regions while the EGF model significantly over-emphasizes rotation-dominant and rotation-production-dominant regions. All three models satisfy the relation between dissipation and the longitudinal velocity derivative variance.

\subsection{Enstrophy and Dissipation}

The probability density distributions (PDFs) of enstrophy and dissipation in isotropic turbulence (Meneveau \& Sreenivasan 1991; Bershadskii et al. 1993; Donzis et al. 2008) provide another useful test for comparing Lagrangian velocity gradient models. Figure 4 compares the dissipation (top) and enstrophy (bottom) PDFs of the RFD (left), EGF (middle), and RDGF (right) models with the DNS results at two $R e_{\lambda}$ values. The RFD model appears to produce exponential tails (straight lines on the log-linear plot) rather than stretched exponential. The EGF model is much improved for the dissipation and enstrophy PDF, appearing somewhat closer to the characteristic stretched exponential shape. The RDGF model provides the best agreement with both dissipation and enstrophy distributions, displaying the stretched-exponential shape for both. It should be kept in mind that the EGF and RDGF do not have explicit Reynolds number dependence. Again, as a qualitative observation, the RDGF model gives results that may appear even more realistic for lower $R e_{\lambda}$.

\subsection{Vorticity and Strain-Rate}

One of the well-known features of velocity gradient statistics in turbulent flows is the non-trivial alignment of the vorticity vector with respect to the three eigenvectors of the strain-rate tensor (Ashurst et al. 1987). The vorticity tends to align more closely with the strain-rate eigenvector associated with the intermediate eigenvalue. Meanwhile, the vorticity tends to be more perpendicular with respect to the strain-rate eigenvector of the smallest eigenvalue. The alignment distribution between the vorticity and the eigenvector of the largest strain-rate eigenvalue tends to be fairly uniform in comparison.

Figure 5a-c shows the PDFs for the cosines of the angles between vorticity and strain-rate eigenvectors. The DNS results at $R e_{\lambda}=430$ are used for comparison here; these statistics show virtually no dependence on $R e_{\lambda}$. All three models mimic the wellknown trend outlined above. The RFD model slightly underpredicts the anti-alignment of vorticity with the smallest strain-rate eigenvalue, while displaying a slight preference toward anti-alignment for the largest eigenvalue. The EGF consistently under-predicts the alignment biases seen in the DNS results. It appears that the RDGF model obtains the best agreement overall. 

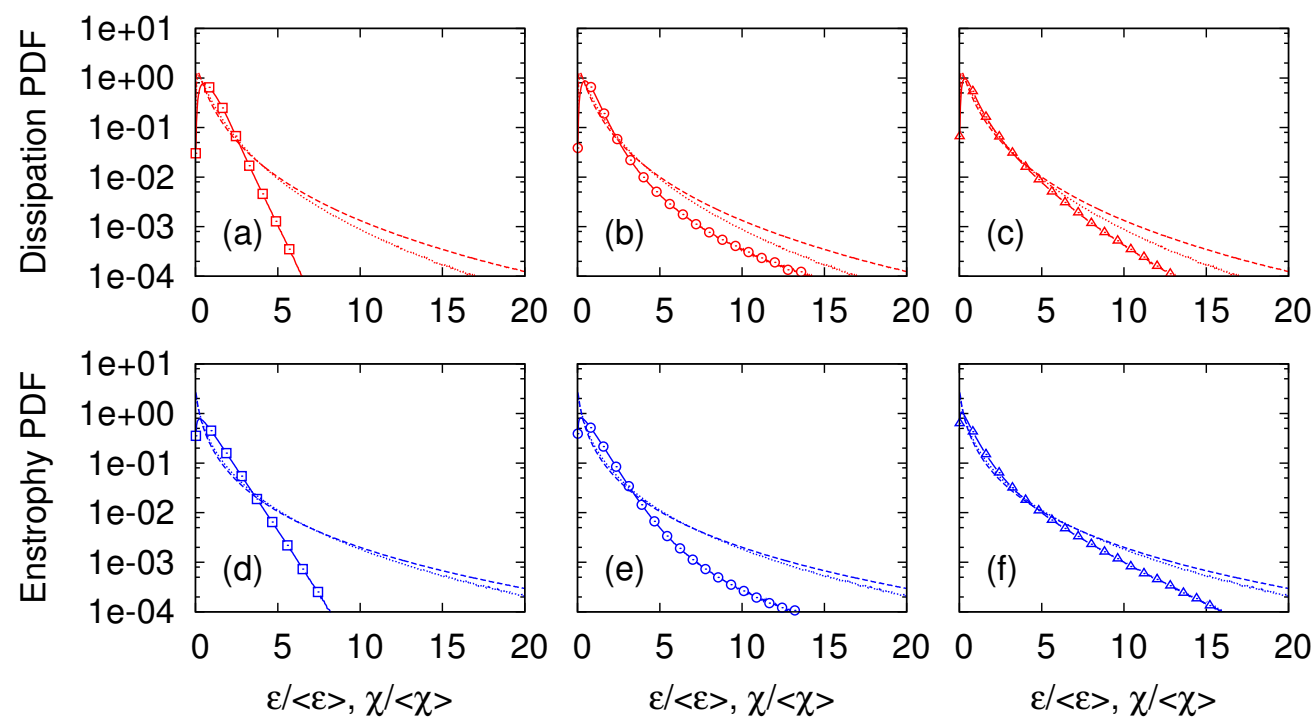

FiguRE 4. PDFs of dissipation (a-c) and enstrophy (d-f) normalized by their mean values for RFD (a,d), EGF (b,e), RDGF (c,f). Solid lines with symbols indicate model results, and DNS results are shown with dashed $\left(R e_{\lambda}=430\right)$ and dotted $\left(R e_{\lambda}=160\right)$ lines.
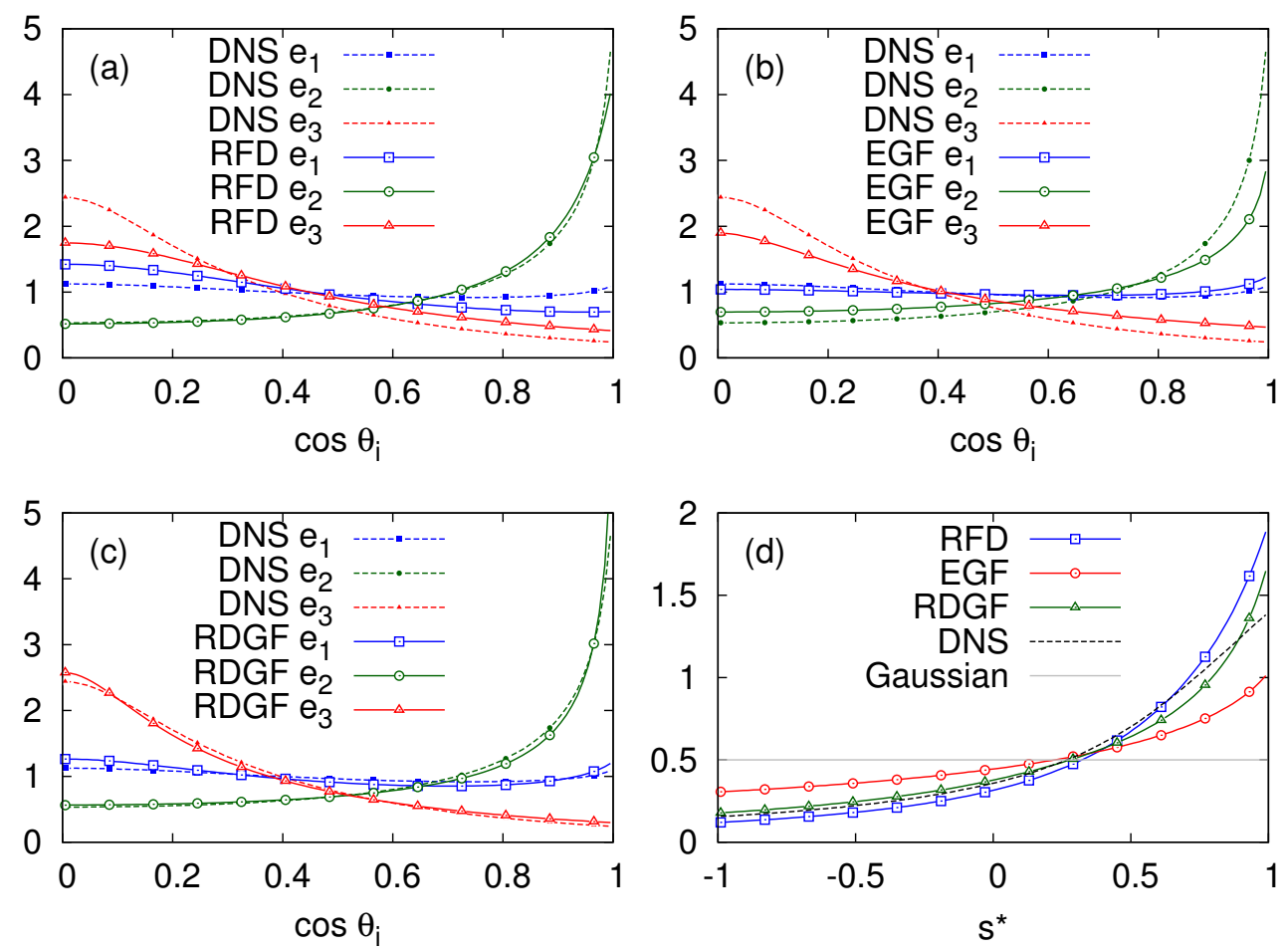

Figure 5. Probability distribution functions for the cosine of the angle between vorticity and the strain-rate eigenvectors: (a) RFD, (b) EGF, (c) RDGF. (d) Probability density functions for $s^{*}$, as defined in (5.1), for the three models compared with DNS results and Gaussian field statistics. 


\section{$\left\langle s^{*}\right\rangle \quad\left\langle\Lambda_{1}\right\rangle \tau_{\eta} \frac{\left\langle\Lambda_{2}\right\rangle}{\left\langle\Lambda_{1}\right\rangle} \quad\left\langle\cos \left(\theta_{1}\right)\right\rangle\left\langle\cos \left(\theta_{2}\right)\right\rangle\left\langle\cos \left(\theta_{3}\right)\right\rangle$}

\begin{tabular}{ccccccc}
\hline RFD & 0.441 & 0.400 & 0.270 & 0.428 & 0.663 & 0.374 \\
EGF & 0.190 & 0.421 & 0.123 & 0.500 & 0.597 & 0.377 \\
RDGF & 0.347 & 0.392 & 0.224 & 0.473 & 0.656 & 0.317 \\
DNS & 0.371 & 0.366 & 0.231 & 0.484 & 0.659 & 0.311
\end{tabular}

TABLE 4. Various mean values for strain-rate and vorticity measures.

Lund \& Rogers (1994) introduced the measure $-1 \leqslant s^{*} \leqslant 1$ using the eigenvalues of the strain-rate tensor,

$$
s^{*}=-\frac{3 \sqrt{6} \Lambda_{1} \Lambda_{2} \Lambda_{3}}{\left(\Lambda_{1}^{2}+\Lambda_{2}^{2}+\Lambda_{3}^{2}\right)^{3 / 2}},
$$

which compares the relative magnitudes of each of the three strain-rate eigenvalues taking into account that they must add up to zero. Figure 5(d) reports the PDFs for the three models considered here, shown in comparison to DNS results $\left(R e_{\lambda}=430\right)$. It is wellknown that turbulent velocity gradients are biased toward $s^{*}>0$, i.e. more distortion toward disk-like fluid elements (Lund \& Rogers 1994; Meneveau 2011). All three models reflect this trend. The RFD model over-predicts the bias toward positive $s^{*}$, while the EGF model under-predicts it. The RDGF model appears to produce results in closest comparison with DNS.

Table 4 compares ensemble averages for some of these vorticity and strain-rate statistics, helping quantify the above discussion. Additionally available from this table is the ratio of average strain-rate eigenvalues, for which the RDGF models also provides good predictions.

\subsection{Dynamics in the $Q-R$ Plane}

Another salient feature of turbulent velocity gradient statistics is the teardrop shaped contours of the joint-probability density function for the $Q$ and $R$ invariants. Figure 6 compares such joint PDFs from the three models with DNS results $\left(R e_{\lambda}=430\right)$. Each model reproduces to some extent the features in the DNS results, most notably the teardrop shape.

The RFD results are too compact, lacking sufficient excursions far from the mean, as also seen previously for the single component PDFs in figure 3. One also observes a less prominent high-probability filament descending down the positive $R$ branch of the Viellefosse line. The EGF model results are more accurate in their depiction of the high probability region along the Viellefosse line but a less realistic aspect of the EGF results is the exaggerated higher-probability in the positive $Q$ region compared to the negative $Q$ region. This feature is evidently responsible for the EGF model's departure from $\langle Q\rangle=0$ (the EGF also does not reproduce $\langle R\rangle=0$ ). The results from the RDGF mapping closure share some of these strengths and weaknesses. For the RDGF, the low probability contours remain too compact, though less so than in the case of the RFD model. The shape of the high-probability regions closely mirrors those for the DNS. Additionally, there is some promising spread for the low-probability contours into the high positive $Q$ regions. However, overall, the details of the low-probability contours (the tails of the joint distribution) still represents a challenge for all three models.

Neglecting the stochastic forcing for the moment, the dynamical equations for $Q$ and 

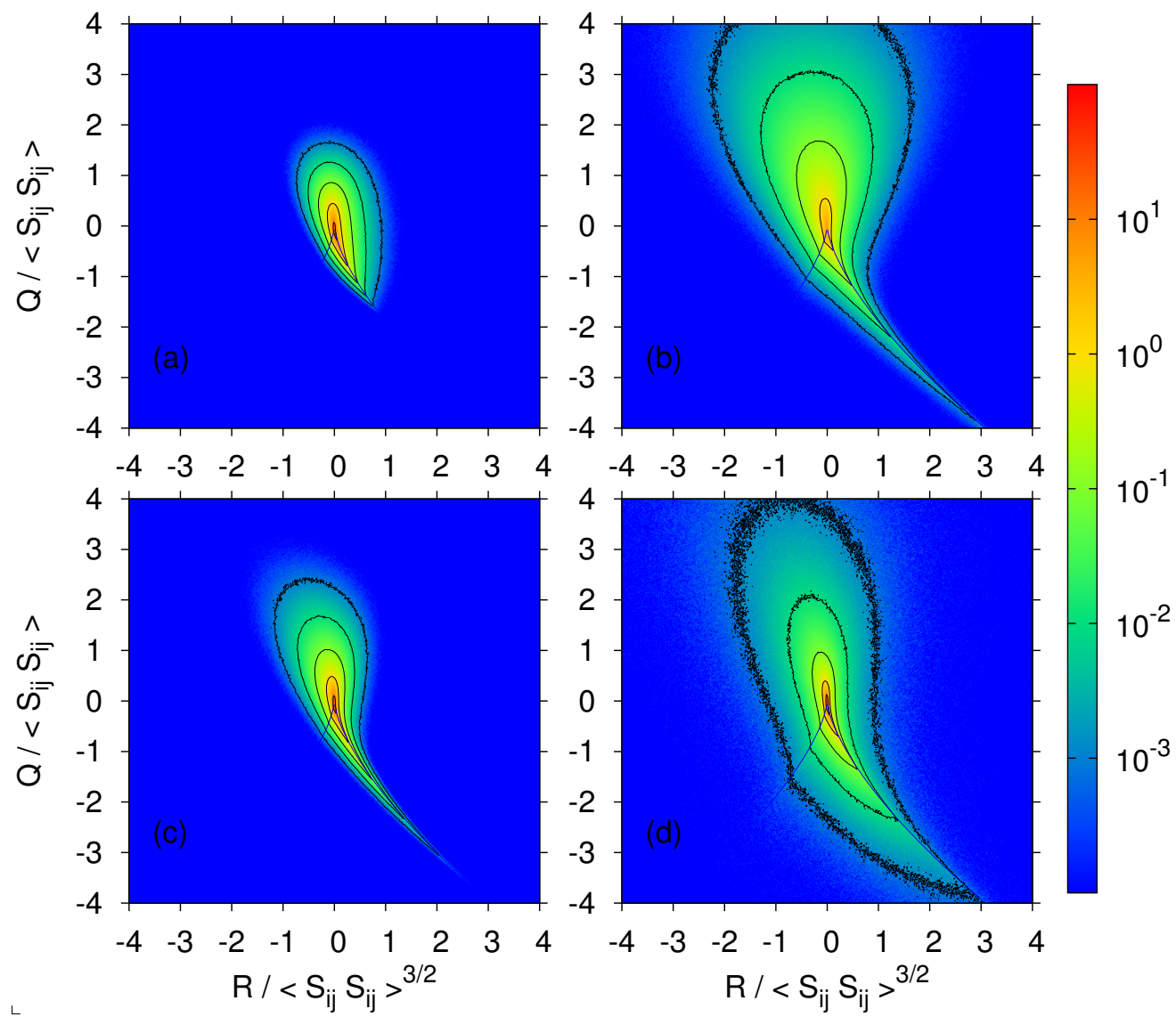

FiguRE 6. Logarithmically scaled joint-probability density function for the invariants $Q$ and $R$ as given by (a) RFD, (b) EGF, (c) RDGF, and (d) DNS.

$R$ are (Chevillard et al. 2008),

$$
\frac{d Q}{d t}=-3 R+A_{i j} P_{j i}^{(d)}-\nu A_{i j} \nabla^{2} A_{j i}, \quad \frac{d R}{d t}=\frac{2}{3} Q^{2}+A_{i j} A_{j k} P_{k i}^{(d)}-\nu A_{i j} A_{j k} \nabla^{2} A_{k i} .
$$

The dynamics in probability space can be recovered thus from conditional averaging,

$$
\begin{gathered}
\left\langle\frac{d Q}{d t} \mid Q, R\right\rangle=-3 R+\left\langle A_{i j} P_{j i}^{(d)} \mid Q, R\right\rangle-\nu\left\langle A_{i j} \nabla^{2} A_{j i} \mid Q, R\right\rangle, \\
\left\langle\frac{d R}{d t} \mid Q, R\right\rangle=\frac{2}{3} Q^{2}+\left\langle A_{i j} A_{j k} P_{k i}^{(d)} \mid Q, R\right\rangle-\nu\left\langle A_{i j} A_{j k} \nabla^{2} A_{k i} \mid Q, R\right\rangle .
\end{gathered}
$$

These equations represent average velocities in the $Q R$ probability space which, when multiplied with the local probability density, represent fluxes in probability space. They are evaluated based on DNS as well as from the three models. In order to compare them under similar conditions, averages are evaluated as an a priori test, by evaluating the model results from an ensemble of DNS trajectories. In practice, we found that the most significant effect of this approach (as opposed to sample the statistics along model evaluations) was to increase the domain in $Q R$ space where the average velocities could be obtained.

Figure 7 shows the $Q R$-space velocities attributed to the pressure Hessian term for 

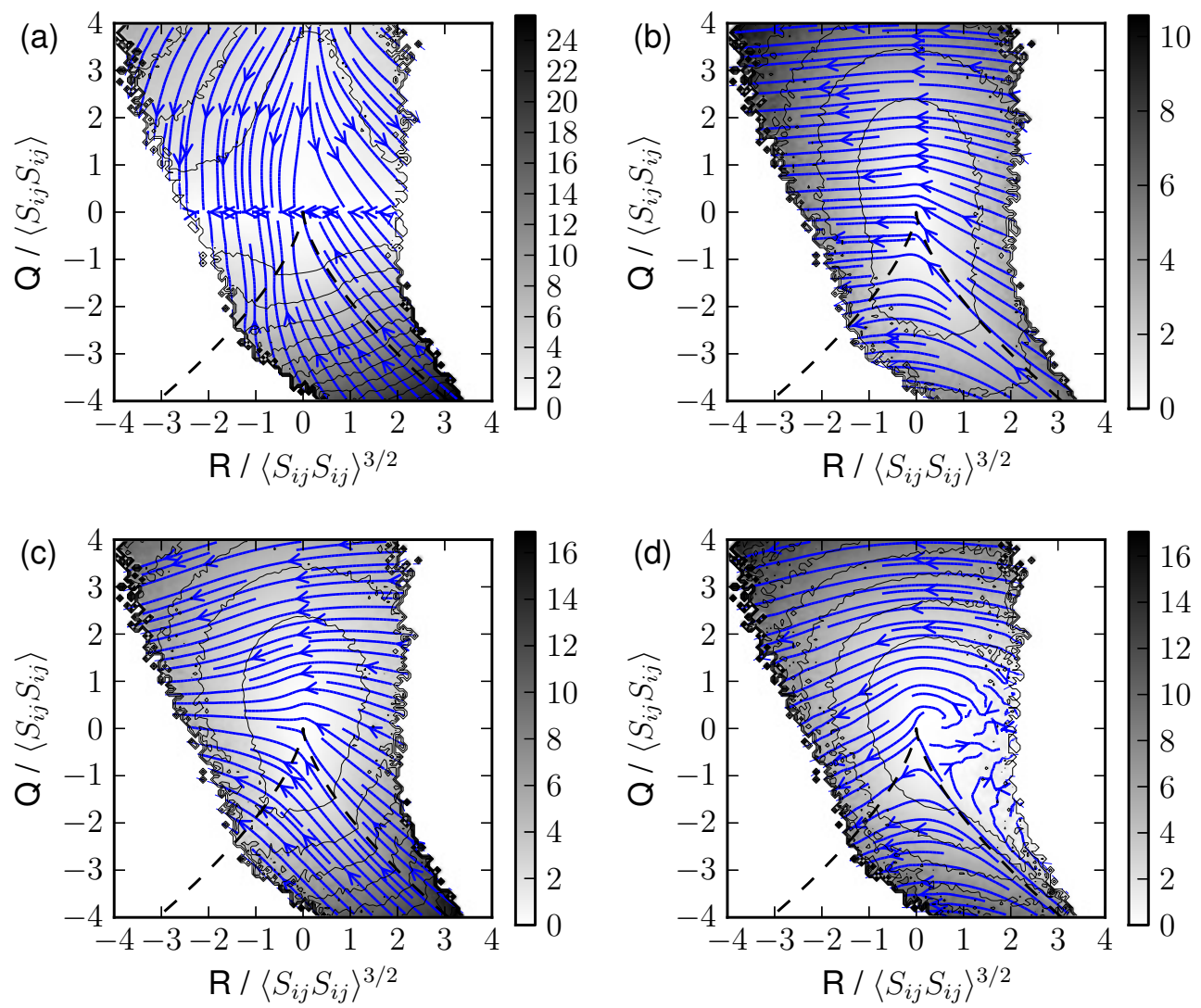

FiguRE 7. Thick lines with arrows represent "streamlines" in the $Q R$-plane due to the deviatoric part of the pressure Hessian. Thin lines represent contours for the velocity magnitude in the $Q R$-plane. Results are as given by (a) RFD, (b) EGF, (c) RDGF mapping closure, and (d) DNS.

the three models compared with DNS results $\left(R e_{\lambda}=430\right)$. The primary action of the RFD pressure Hessian is to oppose the restricted Euler motion along the Vieillefosse tail. In fact, the magnitude of the pressure Hessian opposing the restricted Euler singularity along the Vieillefosse tail is too strong in comparison with the DNS data. As previously noted (Chevillard et al. 2008), the RFD pressure Hessian lacks the right-to-left motion seen in the DNS and the other two models. This elucidates the shortcoming of the upstream isotropic assumption for the pressure Hessian tensor. In fact, it is a significant contribution of the Gaussian form of the pressure Hessian that it adds this right-to-left tendency due to the deviatoric component of the tensor.

The EGF pressure Hessian tends to oppose the singularity with smaller magnitude than the DNS results indicate, while the RDGF opposes with slightly larger magnitude than DNS. While the right-to-left motion is captured by the EGF and RDGF closures, a few more subtle features of the DNS results are not. First, the relatively ambient region of positive $R$ near $Q=0$ has an unphysically active right-to-left motion in the EGF and RDGF closures. Secondly, the DNS results indicate opposition to restricted Euler along the left side of the Vieillefosse line, which is not replicated by the EGF or RDGF closures. Other subtle differences and similarities may be noted, but the above discussion summarizes the most important trends noticeable.

The velocities in $Q R$-space from the viscous Laplacian are shown in figure 8 for each 

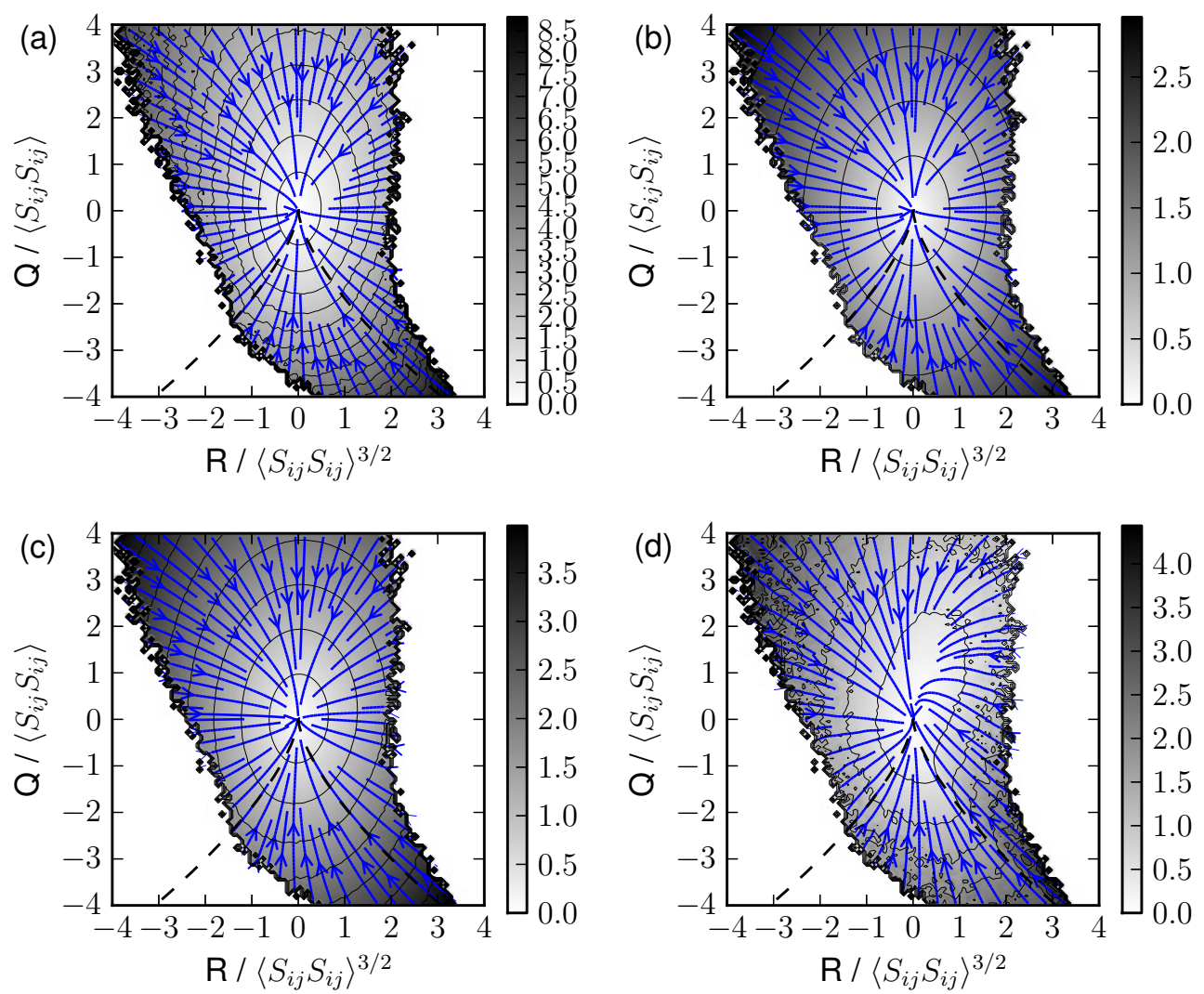

FiguRE 8. Thick lines with arrows represent streamlines in the $Q R$-plane due to the viscous Laplacian. Thin lines represent contours for the velocity magnitude in the $Q R$-plane, non-dimensionalized by powers of $\left\langle S_{i j} S_{i j}\right\rangle$. Results are as given by (a) RFD, (b) EGF, (c) RDGF, and (d) DNS.

of the models compared with DNS. All the models produce the same structure: the viscous Laplacian damps the velocity gradient, thus trajectories are pushed toward the origin in $Q R$-space. Note that the DNS results show some slight deviation from pure damping structure. For example, near $Q=0$ for $R>0$, there is an upward trend in the streamlines instead of proceeding straight toward the origin. Each of the models fail to capture this effect. Thus, updating the upstream conditions of the conditional viscous Hessian produces minimal changes in the behavior of the closure. It appears that the upstream isotropic assumption of RFD model for the viscous term produces relatively more accurate results than was the case for the pressure Hessian.

In terms of magnitude, the RFD model is too strong. The EGF model produces good agreement with DNS in magnitude for the $Q<0, R>0$ region near the Vieillefosse tail, while it is too weak in the $Q>0, R<0$ region. The RDG model has magnitudes in good agreement with DNS for $Q>0, R<0$ but is too strong in the $Q<0, R>0$ along the Viellefosse tail.

The above $Q R$-space analysis shows advantages of the EGF and RDGF closures over the RFD closure. Of particular importance is that the RFD pressure Hessian does not have a strong tendency to decrease $R$. The structure of the deviatoric pressure Hessian from the Gaussian fields provides this effect. Furthermore, the RFD model's 


\begin{tabular}{ccc} 
& $\rho_{\mathbf{P}^{(d)}}$ & $\rho_{\nabla^{2} \mathbf{A}}$ \\
\hline RFD & 0.23 & 0.41 \\
GF & 0.43 & 0.60 \\
EGF & 0.43 & 0.60 \\
RDGF & 0.37 & 0.61
\end{tabular}

TABLE 5. Correlation coefficients for three models with DNS results at $R e_{\lambda}=430$.

over-prediction of magnitude for both of the unclosed terms results in the overly compact joint-PDF contours seen in figure 6 .

\subsection{Correlation Coefficients}

It is interesting to compare the a priori success of each model in terms of correlation coefficients for the deviatoric part of the pressure Hessian and the viscous Laplacian. For the deviatoric part of the pressure Hessian, the correlation coefficient is defined as,

$$
\rho_{\mathbf{P}^{(d)}}=\frac{\left\langle P_{i j}^{(d), \text { DNS }} P_{i j}^{(d), \text { model }}\right\rangle}{\sqrt{\left\langle P_{i j}^{(d), \text { DNS }} P_{i j}^{(d), \text { DNS }}\right\rangle\left\langle P_{i j}^{(d), \text { model }} P_{i j}^{(d), \text { model }}\right\rangle}} .
$$

A similar correlation coefficient is also defined for the viscous Laplacian. These are computed using $8^{t h}$-order finite differencing from an ensemble of 10 million points in the DNS results.

Table 5 shows the resulting correlation coefficients. Included also is the original Gaussian Fields (GF) closure of Wilczek \& Meneveau (2014), which did not provide a statistically stationary solution but rather succumbs to the finite-time singularity similar to the restricted Euler. Overall, the viscous Laplacian models are more successful than the pressure Hessian models. The RFD model has the lowest a-priori correlation coefficients for both closures. The difference between the GF and EGF model in Table 5 is minimal.

The RDGF model actually shows slightly lower correlation for its pressure Hessian model, indicating that the effect of the recent deformation on the Gaussian structure is perhaps not as helpful as one might have hoped. Perhaps the real advantage of the recent deformation is that the magnitude is increased without abandoning the analytical coefficients (i.e. $\alpha=-\frac{2}{7}, \beta=\frac{2}{5}$ ). The effect is that the singularity is avoided without recourse to DNS-tuned coefficients.

\subsection{Computational Cost}

It is useful to mention that these three models are not equal in terms of computational cost. The above results were computed using a Fortran 90 code executed on a single processor. A minimal code involving only time advancement of the velocity gradient tensor without any statistical calculations was timed for the three models. It was found that, per time step, the RFDF model requires about 1.5 times longer than the EGF model, while the RDGF model takes about 2.5 times longer. It is worth noting, however, that the RFD and RDGF models were found to run smoothly and accurately with a time step of about $d t=0.04 \tau_{\eta}$, while the EGF model required a time step of $d t=0.01 \tau_{\eta}$ to avoid singularity. Even with such a small time step, the stochastic system exhibited rare rogue trajectories that had an overwhelming effect on the flatness factors, preventing convergence in a reasonable amount of time (e.g. trajectories advanced for $1000 \tau_{\eta}$ ). We 
note that Wilczek \& Meneveau (2014) used an even smaller time step of $d t=0.001 \tau_{\eta}$. Therefore, the computational cost advantage of EGF model is not realized. The RFD model does have a computation cost per time step approximately $40 \%$ smaller than that of the RDGF model.

\section{Conclusions}

In this paper, a new closure, the Recent Deformation of Gaussian Fields (RDGF) mapping closure, for the pressure Hessian and viscous Laplacian along Lagrangian trajectories in turbulent flow is introduced. The new closure benefits from the insights of both the Recent Fluid Deformation (RFD) and Gaussian Field (GF) closures. The GF closure calculations are applied for the initial upstream conditions of the conditional pressure Hessian and viscous Laplacian, before performing a recent fluid deformation mapping to complete the closure. The coefficients for Gaussian fields can be used and three remaining free parameters related to forcing and time-scale are constrained so that the model reproduces known exact statistical relations. The stochastic forcing for this model is also generalized from that used for the previous models so that the magnitude of the symmetric and anti-symmetric forcings can be applied independently.

A priori evaluation of the models in terms of correlation coefficients and $Q R$-space velocities reveals the shortcomings of RFD closure: the magnitudes of the unclosed terms are significantly over-estimated, and the role of the pressure Hessian in decreasing the $R$ invariant is absent. These shortcomings are much improved using the conditional pressure Hessian from Gaussian fields. On the other hand, the exponential non-linearity of the recent deformation tensor allows for more effective prevention of singularities. As a result, the RDGF model does not require DNS-tuned coefficients in order to prevent the singularity. In this way, the RDGF model has the robustness and analytical closedness of RFD model while providing a more realistic structure of the pressure Hessian from the GF closure.

A comparison of various single-time statistics suggests that the RDGF model can provide excellent results in comparison to the two previous models. However, by comparison with DNS at $R e_{\lambda}=430$, the quantitative results reveal remaining shortcomings such as lack of increasing long tails and intermittency. The RDGF results seem more consistent with lower Reynolds number DNS results. This highlights one of the major limitations of the current model, that it does not include a robust way of changing the Reynolds number whereas velocity gradient statistics are known to depend strongly on Reynolds number. The RFD model does include a mechanism for increasing the Reynolds number, but only in a very limited range. In fact, RFD applied for $R e_{\lambda} \approx 430$ is already outside the range where it performs well. The RDGF mapping closure suffers these same drawbacks as RFD, even if the skewness factor is adjusted to reflect its (weak) dependence on Reynolds number.

In summary, this paper builds a new closure framework for the conditional pressure Hessian and viscous Laplacian which leverages insights of previous approaches. It provides, therefore, a promising direction for future investigations of velocity gradient statistics in isotropic turbulence. At sufficiently high Reynolds numbers, where approximate isotropy of small scales is a safe assumption, models for isotropic turbulence can be applicable for a more general class of turbulent flows, for which some applications may find efficient access to velocity gradient statistics useful. 


\section{Acknowledgments}

PJ was supported by a National Science Foundation Graduate Research Fellowship Program under Grant No. DGE-1232825, and CM by a grant from the NSF CBET1507469. PJ thanks Michael Wilczek for many insightful discussions during his time at Johns Hopkins.

\section{Appendix A. Isotropic Tensorial Stochastic Forcing for Symmetric and Anti-Symmetric Components}

In this appendix, the form of the stochastic forcing in (2.7) is established. As identified in the text, the forcing should have the form $d F_{i j}=b_{i j k \ell} d W_{k \ell}$, and can be thought of as a sum of symmetric and anti-symmetric forcing, $d F_{i j}=d F_{i j}^{(s)}+d F_{i j}^{(a)}$, where $d F_{i j}^{(s)}=\frac{1}{2}\left(d F_{i j}+d F_{j i}\right)$ and $d F_{i j}^{(a)}=\frac{1}{2}\left(d F_{i j}-d F_{j i}\right)$. Since $d W_{i j}$ represents a tensorial Wiener process, i.e. $\left\langle W_{i j}\right\rangle=0$ and $\left\langle d W_{i j} d W_{k \ell}\right\rangle=\delta_{i k} \delta_{j \ell} d t$, then

$$
\left\langle d F_{i j} d F_{k \ell}\right\rangle=b_{i j m n} b_{k \ell m n} d t=D_{i j k \ell} d t .
$$

Therefore, the forcing contributes a variance growth rate of

$$
d\left\langle F_{i j} F_{i j}\right\rangle=\left\langle d F_{i j} d F_{i j}\right\rangle=D_{i j i j} d t
$$

and furthermore, the symmetric and anti-symmetric variance growth rates are,

$$
\begin{aligned}
& d\left\langle F_{i j}^{(s)} F_{i j}^{(s)}\right\rangle=\left\langle d F_{i j}^{(s)} d F_{i j}^{(s)}\right\rangle=\frac{1}{2}\left(D_{i j i j}+D_{i j j i}\right) d t \equiv D_{s} d t . \\
& d\left\langle F_{i j}^{(a)} F_{i j}^{(a)}\right\rangle=\left\langle d F_{i j}^{(a)} d F_{i j}^{(a)}\right\rangle=\frac{1}{2}\left(D_{i j i j}-D_{i j j i}\right) d t=D_{a} d t .
\end{aligned}
$$

Here, by definition, $D_{s}$ and $D_{a}$ represent the growth rate of the variance of symmetric and anti-symmetric parts of the forcing.

To model isotropic turbulence, the stochastic forcing should be statistically isotropic. The most general isotropic form for the diffusion tensor is

$$
D_{i j k \ell}=d_{1} \delta_{i j} \delta_{k \ell}+d_{2} \delta_{i k} \delta_{j \ell}+d_{3} \delta_{i \ell} \delta_{j k} .
$$

Requiring also that the forcing be trace-free (incompresibility), then

$$
D_{i i k \ell}=\left(3 d_{1}+d_{2}+d_{3}\right) \delta_{k \ell}=0 .
$$

Combining this constraint with the two definitions of $D_{s}$ and $D_{a}$ given above,

$$
\begin{gathered}
D_{s}=\frac{1}{2}\left(D_{i j i j}+D_{i j j i}\right)=3 d_{1}+6 d_{2}+6 d_{3}, \\
D_{a}=\frac{1}{2}\left(D_{i j i j}-D_{i j j i}\right)=3 d_{2}-3 d_{3},
\end{gathered}
$$

then the system of 3 equations and 3 unknowns can be solved for

$$
D_{i j k \ell}=-\frac{D_{s}}{15} \delta_{i j} \delta_{k \ell}+\left(\frac{D_{s}}{10}+\frac{D_{a}}{6}\right) \delta_{i k} \delta_{j \ell}+\left(\frac{D_{s}}{10}-\frac{D_{a}}{6}\right) \delta_{i \ell} \delta_{j k} .
$$

The choice of $D_{s}=D_{a}=15$ reduces to the form of Chevillard and Meneveau (Chevillard et al. 2008) used for the RFD model,

$$
D_{i j k \ell}=-\delta_{i j} \delta_{k \ell}+4 \delta_{i k} \delta_{j \ell}-\delta_{i \ell} \delta_{j k}
$$


To implement this forcing, however, the tensor $b_{i j k \ell}$ is necessary, thus the equation $b_{i j m n} b_{k \ell m n}=D_{i j k \ell}$ must be solved. Using the general isotropic form

$$
b_{i j k \ell}=b_{1} \delta_{i j} \delta_{k \ell}+b_{2} \delta_{i k} \delta_{j \ell}+b_{3} \delta_{i \ell} \delta_{j k}
$$

the tensor contractions yield the following system of equations,

$$
\begin{gathered}
d_{1}=3 b_{1}^{2}+2 b_{1} b_{2}+2 b_{1} b_{3}=-\frac{D_{s}}{15}, \\
d_{2}=b_{2}^{2}+b_{3}^{2}=\frac{D_{s}}{10}+\frac{D_{a}}{6}, \\
d_{3}=2 b_{2} b_{3}=\frac{D_{s}}{10}-\frac{D_{a}}{6} .
\end{gathered}
$$

Solution to this system of equations yields,

$$
b_{i j k \ell}=-\frac{1}{3} \sqrt{\frac{D_{s}}{5}} \delta_{i j} \delta_{k \ell}+\frac{1}{2}\left(\sqrt{\frac{D_{s}}{5}}+\sqrt{\frac{D_{a}}{3}}\right) \delta_{i k} \delta_{j \ell}+\frac{1}{2}\left(\sqrt{\frac{D_{s}}{5}}-\sqrt{\frac{D_{a}}{3}}\right) \delta_{i \ell} \delta_{j k},
$$

which reduces to the form of Chevillard et al. (2008) with the choice $D_{s}=D_{a}=15$. Meanwhile, Wilczek \& Meneveau (2014) tuned $D_{s}=D_{a}$ such that the definition of $\tau_{\eta}$ was consistent between model and numerics. In the authors' current view, e.g. considering (2.8) and (2.9), there is no a-priori reason that the strain-rate and vorticity should be forced stocastically with the same amplitude, therefore, the present model considers $D_{s}$ and $D_{a}$ to be two independent tuning parameters.

\section{Appendix B. Analytical Calculation of $\gamma$ for the Gaussian Fields Representation of the Conditional Pressure Hessian}

A key component to both the Enhanced Gaussian closure and the recent-deformation of Gaussian fields mapping closure is the representation of a conditional pressure Hessian using (2.20). While the coefficients $\alpha$ and $\beta$ were directly evaluated from the Gaussian fields closure, the last coefficient is determined by the details of the longitudinal correlation function, (2.21). Calculations are easier using the longitudinal structure function $D_{L L}(r)=2 \overline{u^{2}}(1-f(r))$.

$$
\gamma=\frac{6}{25}+\frac{16}{75 D_{L L}^{\prime \prime}(0)^{2}} \int_{0}^{\infty} \frac{D_{L L}^{\prime}(r) D_{L L}^{\prime \prime \prime}(r)}{r} d r
$$

where $D_{L L}^{\prime \prime}(0)=\frac{2 \epsilon}{15 \nu}$ according to the proper viscous range behavior. Using the approach of Batchelor (1951), the viscous and inertial range behavior of the structure function can be preserved using a blending function,

$$
D_{L L}(r)=C_{2} \epsilon^{2 / 3} r^{2 / 3} F\left(\frac{r}{\gamma_{2} \eta}\right)
$$

Here, we assume K41 scaling for the inertial range with Kolmogorov coefficient $C_{2} \approx 2.0$ (Pope 2000). The blending function of Batchelor (1951) is

$$
F\left(\frac{r}{\gamma_{2} \eta}\right)=\left[1+\left(\frac{r}{\gamma_{2} \eta}\right)^{-2}\right]^{-2 / 3}
$$




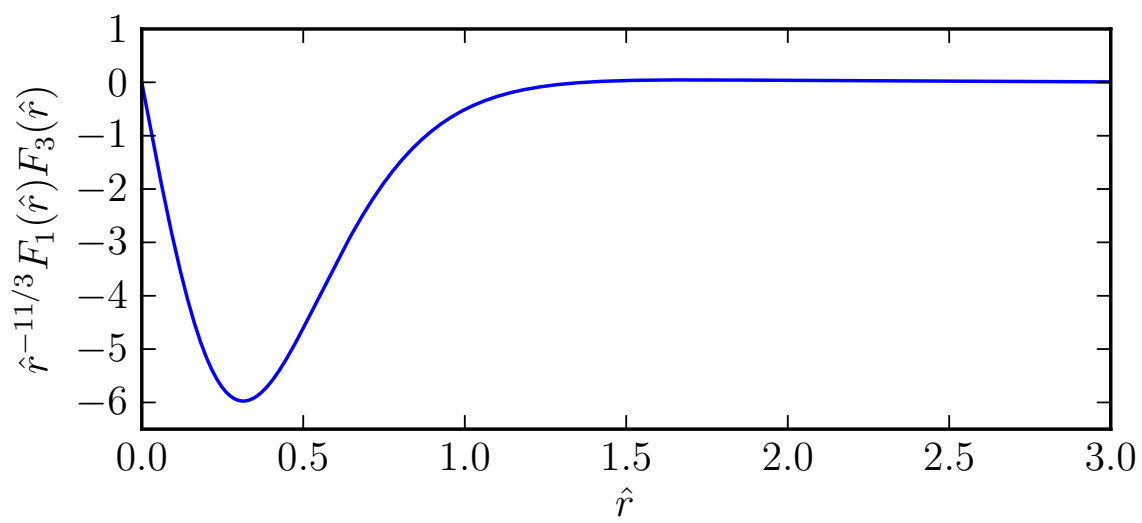

FIGURE 9. Integrand in (B 7) plotted in normalized variables $\hat{r}=\frac{r}{\gamma_{2} \eta}$ with $\gamma_{2} \approx 13$.

where $\gamma_{2}=\left(15 C_{2}\right)^{3 / 4} \approx 13$ sets the cross-over point between viscous and inertial behavior, recovering the correct viscous range behavior. With the application of product rule differentiation, we can write

$$
\begin{aligned}
& D_{L L}^{\prime}(r)=C_{2} \epsilon^{2 / 3} r^{-1 / 3} F_{1}\left(\frac{r}{\gamma_{2} \eta}\right), \\
& D_{L L}^{\prime \prime \prime}(r)=C_{2} \epsilon^{2 / 3} r^{-7 / 3} F_{3}\left(\frac{r}{\gamma_{2} \eta}\right),
\end{aligned}
$$

and thus the integral simplifies under the change of variable $\hat{r}=\frac{r}{\gamma_{2} \eta}$,

$$
\gamma=\frac{6}{25}+\frac{12}{225} I
$$

where

$$
I=\int_{0}^{\infty} \hat{r}^{-11 / 3} F_{1}(\hat{r}) F_{3}(\hat{r}) d \hat{r},
$$

with the derivative functions

$$
\begin{gathered}
F_{1}(\hat{r})=\frac{2}{3} F(\hat{r})+\hat{r} F^{\prime}(\hat{r}), \\
F_{3}(\hat{r})=\frac{8}{27} F(\hat{r})-\frac{2}{3} \hat{r} F^{\prime}(\hat{r})+2 \hat{r}^{2} F^{\prime \prime}(\hat{r})+\hat{r}^{3} F^{\prime \prime \prime}(\hat{r}) .
\end{gathered}
$$

This integrand is plotted in figure 9, from which it is apparent that the integral is dominated by contributions from the viscous range, i.e. $r<13 \eta$. Without considering the details of the integration, the manipulation so far shows that $\gamma$ is (approximately) independent of $R e_{\lambda}$ (neglecting weak $R e_{\lambda}$-effects on the cutoff scale), and its precise value is difficult to determine because it will depend heavily on the details of the blending function used. 
The integral can be written fully as

$$
\begin{aligned}
I=\int_{0}^{\infty}\left[\frac{16}{81}(1+\right. & \left.\hat{r}^{2}\right)^{-4 / 3}+\frac{416}{81}\left(1+\hat{r}^{2}\right)^{-7 / 3}-\frac{304}{27}\left(1+\hat{r}^{2}\right)^{-10 / 3} \\
& \left.-\frac{2080}{81}\left(1+\hat{r}^{2}\right)^{-13 / 3}+\frac{2560}{81}\left(1+\hat{r}^{2}\right)^{-16 / 3}\right] \frac{d \hat{r}}{\hat{r}} .
\end{aligned}
$$

To integrate, add

$$
\left(\frac{16}{81}+\frac{416}{81}-\frac{304}{27}-\frac{2080}{81}+\frac{2560}{81}\right) \frac{\left(1+\hat{r}^{2}\right)^{-1 / 3}}{\hat{r}}=0
$$

to the integrand and use the change of variables,

$$
\zeta=1+\hat{r}^{2}, \quad \frac{d \zeta}{2(\zeta-1)}=\frac{d \hat{r}}{\hat{r}} .
$$

As a result, the integral becomes,

$$
\begin{gathered}
I=\frac{1}{2} \int_{1}^{\infty}\left[-\frac{16}{81} \zeta^{-4 / 3}-\frac{416}{81} \zeta^{-7 / 3}(\zeta+1)+\frac{304}{27} \zeta^{-10 / 3}\left(\zeta^{2}+\zeta+1\right)\right. \\
\left.+\frac{2080}{81} \zeta^{-13 / 3}\left(\zeta^{3}+\zeta^{2}+\zeta+1\right)-\frac{2560}{81} \zeta^{-16 / 3}\left(\zeta^{4}+\zeta^{3}+\zeta^{2}+\zeta+1\right)\right] d \zeta .
\end{gathered}
$$

Then algebraic simplification

$$
I=\frac{1}{2} \int_{1}^{\infty}\left[\frac{16}{81} \zeta^{-7 / 3}+\frac{432}{81} \zeta^{-10 / 3}-\frac{480}{81} \zeta^{-13 / 3}-\frac{2560}{81} \zeta^{-16 / 3}\right] d \zeta,
$$

and completing the power-law integrations results in

$$
I=-\frac{302}{91}
$$

Substitution of this results leads to

$$
\gamma=\frac{86}{1365} \approx 0.063
$$

\section{Appendix C. Gaussian Fields Approximation for the Conditional Hessian of the Velocity Gradient}

This appendix details the derivation of (3.3) in the main text, following the method outlined in Wilczek \& Meneveau (2014). The characteristic function of the turbulent velocity field,

$$
\phi^{u}[\lambda(\mathbf{x})]=\left\langle\exp \left(i \int \lambda_{i}(\mathbf{x}) u_{i}(\mathbf{x}) d \mathbf{x}\right)\right\rangle,
$$

contains all the statistical information necessary to compute the desired conditional mean, namely $\left\langle\frac{\partial^{2} A_{i j}}{\partial x_{p} \partial x_{q}} \mid \mathcal{A}\right\rangle$. To make progress analytically, the turbulent velocity field is taken to be Gaussian, meaning that all $n$-point pdfs are joint-Gaussian,

$$
\phi^{u}[\lambda(\mathbf{x})]=\exp \left(-\frac{1}{2} \iint \lambda_{i}(\mathbf{x}) B_{i j}\left(\mathbf{x}, \mathbf{x}^{\prime}\right) \lambda_{j}\left(\mathbf{x}^{\prime}\right) d \mathbf{x} d \mathbf{x}^{\prime}\right),
$$


where $B_{i j}$ is the two-point covariance tensor, which for homogeneous isotropic turbulence depends only on the separation vector, $\mathbf{r}=\mathbf{x}-\mathbf{x}^{\prime}$, and has the form

$$
B_{i j}\left(\mathbf{x}, \mathbf{x}^{\prime}\right)=B_{i j}(\mathbf{r})=\left\langle u_{1}^{2}\right\rangle\left[f(r) \delta_{i j}+\frac{1}{2} r f^{\prime}(r)\left(\delta_{i j}-\hat{r}_{i} \hat{r}_{j}\right)\right],
$$

where $r=|\mathbf{r}|$ and $\hat{r}_{i}=\frac{r_{i}}{r}$. In this way the characteristic functional, when assumed Gaussian for isotropic turbulence, is uniquely specified by the longitudinal velocity correlation function,

$$
f(r)=\frac{\left\langle u_{1}(\mathbf{x}) u_{1}\left(\mathbf{x}+r \mathbf{e}_{1}\right)\right\rangle}{\left\langle u_{1}^{2}\right\rangle} .
$$

With integration by parts, the relationship between the characteristic functional for the velocity field and that of the velocity gradient field can be shown to be

$$
\phi^{A}[\Lambda]=\phi^{u}[-\nabla \cdot \Lambda]
$$

Again, with integration by parts, substituting this relationship into the Gaussian characteristic functional for the velocity field,

$$
\phi^{A}[\Lambda(\mathbf{x})]=\exp \left(-\frac{1}{2} \iint \Lambda_{i k}(\mathbf{x}) C_{i j k \ell}\left(\mathbf{x}, \mathbf{x}^{\prime}\right) \Lambda_{j \ell}\left(\mathbf{x}^{\prime}\right) d \mathbf{x} d \mathbf{x}^{\prime}\right),
$$

where

$$
C_{i j k \ell}\left(\mathbf{x}, \mathbf{x}^{\prime}\right)=\frac{\partial^{2} B_{i j}}{\partial x_{k} \partial x_{\ell}^{\prime}}\left(\mathbf{x}, \mathbf{x}^{\prime}\right)=\left\langle A_{i k}(\mathbf{x}) A_{j \ell}\left(\mathbf{x}^{\prime}\right)\right\rangle,
$$

is the covariance tensor for the velocity gradient, which only depends on $\mathbf{r}=\mathbf{x}-\mathbf{x}^{\prime}$. It is computed from the Hessian of the velocity covariance tensor,

$$
\begin{aligned}
C_{i j k \ell}(\mathbf{r})= & -\frac{\partial^{2} B_{i j}}{\partial r_{k} \partial r_{\ell}}=\left\langle u_{1}^{2}\right\rangle\left[\left(-\frac{3}{2} \frac{f^{\prime}(r)}{r}-\frac{1}{2} f^{\prime \prime}(r)\right)\left(\delta_{i j} \delta_{k \ell}\right)+\left(\frac{1}{2} \frac{f^{\prime}(r)}{r}\right)\left(\delta_{i k} \delta_{j \ell}+\delta_{i \ell} \delta_{j k}\right)\right. \\
& +\left(\frac{3}{2} \frac{f^{\prime}(r)}{r}-\frac{3}{2} f^{\prime \prime}(r)-\frac{1}{2} r f^{\prime \prime \prime}(r)\right)\left(\delta_{i j} \hat{r}_{k} \hat{r}_{\ell}\right)+ \\
& +\left(-\frac{1}{2} \frac{f^{\prime}(r)}{r}+\frac{1}{2} f^{\prime \prime}(r)\right)\left(\delta_{i \ell} \hat{r}_{j} \hat{r}_{k}+\delta_{k \ell} \hat{r}_{i} \hat{r}_{j}+\delta_{j \ell} \hat{r}_{i} \hat{r}_{k}+\delta_{i k} \hat{r}_{j} \hat{r}_{\ell}+\delta_{j k} \hat{r}_{i} \hat{r}_{\ell}\right) \\
& \left.\left(\frac{3}{2} \frac{f^{\prime}(r)}{r}-\frac{3}{2} f^{\prime \prime}(r)+\frac{1}{2} r f^{\prime \prime \prime}(r)\right)\left(\hat{r}_{i} \hat{r}_{j} \hat{r}_{k} \hat{r}_{\ell}\right)\right] .
\end{aligned}
$$

The desired statistical quantity in this exercise is

$$
\nu\left\langle\frac{\partial^{2} A_{i j}}{\partial x_{k} \partial x_{\ell}} \mid \mathcal{A}\right\rangle=\nu \lim _{r \rightarrow 0} \frac{\partial^{2}}{\partial r_{k} \partial r_{\ell}}\left\langle A_{i j}(\mathbf{x}+\mathbf{r}) \mid \mathcal{A}(\mathbf{x})\right\rangle .
$$

Following exactly the steps outlined in Appendix B2 of Wilczek \& Meneveau (2014),

$$
\left\langle A_{i j}(\mathbf{x}+\mathbf{r}) \mid \mathcal{A}(\mathbf{x})\right\rangle=C_{i k j \ell}(\mathbf{r}) C_{k m \ell n}^{-1}(\mathbf{0}) \mathcal{A}_{m n},
$$

where equality at the origin means

$$
C_{k m \ell n}^{-1}(\mathbf{0})=\frac{2}{15\left\langle u_{1}^{2}\right\rangle f^{\prime \prime}(0)}\left(-4 \delta_{k m} \delta_{\ell n}-\delta_{k n} \delta_{m \ell}\right),
$$

see Appendix B1 of Wilczek \& Meneveau (2014) for details. Combining expressions,

$$
\nu\left\langle\frac{\partial^{2} A_{i j}}{\partial x_{k} \partial x_{\ell}} \mid \mathcal{A}\right\rangle=\nu \lim _{r \rightarrow 0} \frac{\partial^{2} C_{i k j \ell}}{\partial r_{p} \partial r_{q}} C_{k m \ell n}^{-1}(\mathbf{0}) \mathcal{A}_{m n} .
$$


A tedious calculation by twice taking the gradient of (C 8) results in,

$$
\begin{array}{r}
\lim _{r \rightarrow 0} \frac{\partial^{2} C_{i k j \ell}}{\partial r_{p} \partial r_{q}}=\left\langle u_{1}^{2}\right\rangle f^{(4)}(0)\left[-\left(\delta_{i k} \delta_{j \ell} \delta_{p q}+\delta_{i k} \delta_{j p} \delta_{\ell q}+\delta_{i k} \delta_{j q} \delta_{\ell p}\right)\right. \\
+\frac{1}{6}\left(\delta_{i j} \delta_{k \ell} \delta_{p q}+\delta_{i \ell} \delta_{k j} \delta_{p q}+\delta_{i \ell} \delta_{k p} \delta_{j q}+\delta_{i \ell} \delta_{j p} \delta_{k q}+\delta_{i p} \delta_{k \ell} \delta_{j q}+\delta_{i q} \delta_{k \ell} \delta_{j p}\right. \\
\left.\left.+\delta_{j \ell} \delta_{k p} \delta_{i q}+\delta_{j \ell} \delta_{i p} \delta_{k q}+\delta_{i j} \delta_{k p} \delta_{\ell q}+\delta_{i j} \delta_{\ell p} \delta_{k q}+\delta_{k j} \delta_{i p} \delta_{\ell q}+\delta_{k j} \delta_{\ell p} \delta_{i q}\right)\right]
\end{array}
$$

Substitution of (C 11) and (C 13) into (C 12), followed by a tedious calculation of tensor contractions yields,

$$
\begin{aligned}
& \nu\left\langle\frac{\partial^{2} A_{i j}}{\partial x_{k} \partial x_{\ell}} \mid \mathcal{A}\right\rangle=\frac{2 \nu f^{(4)}(0)}{15 f^{\prime \prime}(0)}\left[\left(\frac{23}{6} \mathcal{A}_{i j}+\frac{1}{3} \mathcal{A}_{j i}\right) \delta_{p q}+\left(\frac{23}{6} \mathcal{A}_{i q}+\frac{1}{3} \mathcal{A}_{q i}\right) \delta_{j p}\right. \\
& +\left(\frac{23}{6} \mathcal{A}_{i p}+\frac{1}{3} \mathcal{A}_{p i}\right) \delta_{j q}-\left(\frac{5}{6} \mathcal{A}_{j q}+\frac{5}{6} \mathcal{A}_{q j}\right) \delta_{i p} \\
& \left.-\left(\frac{5}{6} \mathcal{A}_{j p}+\frac{5}{6} \mathcal{A}_{p j}\right) \delta_{i q}-\left(\frac{5}{6} \mathcal{A}_{p q}+\frac{5}{6} \mathcal{A}_{q p}\right) \delta_{i j}\right]
\end{aligned}
$$

which can be written in the form of (3.3) with (3.4).

\section{Appendix D. Determination of $\delta$ Using the Enstrophy Balance}

Using the result of Appendix C, the back-in-time velocity gradient Hessian is given by

$$
\nu\left\langle\frac{\partial^{2} A_{i j}}{\partial X_{p} \partial X_{q}} \mid \mathcal{A}\right\rangle=\delta\left(T_{i j} \delta_{p q}+T_{i q} \delta_{j p}+T_{i p} \delta_{j q}-\frac{2}{21} S_{j q} \delta_{i p}-\frac{2}{21} S_{j p} \delta_{i q}-\frac{2}{21} S_{p q} \delta_{i j}\right),
$$

where the coefficient $\delta$ can be written in terms of the enstrophy dissipation,

$$
\delta=\nu \frac{7}{3} \frac{f^{(4)}(0)}{f^{\prime \prime}(0)}=-\tau_{\eta}^{2} \nu\left\langle\frac{\partial \omega_{i}}{\partial X_{j}} \frac{\partial \omega_{i}}{\partial X_{j}}\right\rangle .
$$

Note that since the Gaussian fields evaluation is back-in-time, so this can be interpreted as the back-in-time enstrophy dissipation. By definition, the RFD-style mapping used to generate the approximate back-in-time values keeps velocity gradients constant, but not velocity Hessians. Therefore, the enstrophy production $\left\langle\omega_{i} S_{i j} \omega_{j}\right\rangle$ is constant under the mapping but the enstrophy dissipation is not constant. Two choices are thus available: apply the enstrophy balance for the back-in-time enstrophy dissipation, or try to invert the mapping effect on the enstrophy dissipation to apply the balance at the present time. It is the opinion of the authors that the second option is desirable, since it leads to the application of the enstrophy balance at the present time rather than back-in-time.

Thus, by modeling choice, the relevant enstrophy balance is

$$
\left\langle\omega_{i} S_{i j} \omega_{j}\right\rangle=\nu\left\langle\frac{\partial \omega_{i}}{\partial x_{j}} \frac{\partial \omega_{i}}{\partial x_{j}}\right\rangle
$$

To map the enstrophy dissipation forward in time,

$$
\left\langle\frac{\partial \omega_{i}}{\partial X_{j}} \frac{\partial \omega_{i}}{\partial X_{j}}\right\rangle=\left\langle\frac{\partial x_{k}}{\partial X_{j}} \frac{\partial \omega_{i}}{\partial x_{k}} \frac{\partial \omega_{i}}{\partial x_{\ell}} \frac{\partial x_{\ell}}{\partial X_{j}}\right\rangle=\left\langle C_{k \ell} \frac{\partial \omega_{i}}{\partial x_{k}} \frac{\partial \omega_{i}}{\partial x_{\ell}}\right\rangle \approx C_{k \ell}\left\langle\frac{\partial \omega_{i}}{\partial x_{k}} \frac{\partial \omega_{i}}{\partial x_{\ell}}\right\rangle .
$$

In the last step, the value of $C_{k \ell}$ is localized by approximation, so that no ensemble averages are needed to advance the model stochastic equations. Finally, the enstrophy 
dissipation tensor is assumed isotropic,

$$
\left\langle\frac{\partial \omega_{i}}{\partial x_{k}} \frac{\partial \omega_{i}}{\partial x_{\ell}}\right\rangle \approx \frac{1}{3}\left\langle\frac{\partial \omega_{i}}{\partial x_{j}} \frac{\partial \omega_{i}}{\partial x_{j}}\right\rangle \delta_{k \ell} .
$$

Substituting, the resulting enstrophy balance is

$$
\left\langle\omega_{i} S_{i j} \omega_{j}\right\rangle=\frac{3 \nu}{C_{k k}}\left\langle\frac{\partial \omega_{i}}{\partial X_{j}} \frac{\partial \omega_{i}}{\partial X_{j}}\right\rangle .
$$

Using the isotropic relation $\left\langle\omega_{i} S_{i j} \omega_{j}\right\rangle=-\frac{7 \mathcal{S}}{6 \sqrt{15} \tau_{\eta}^{3}}$ on the left side and the definition of $\delta$ in terms of enstrophy dissipation on the right side, the result is

$$
\delta=\frac{C_{k k}}{3} \frac{7}{6 \sqrt{15}} \frac{\mathcal{S}}{\tau_{\eta}} .
$$

The result given by Wilczek \& Meneveau (2014) is recovered when the mapping is removed, $D_{i j}=\delta_{i j}$, so that $C_{k k}=3$. In this way, the $\delta$ coefficient itself depends on the recent deformation. This provides the convenience of an additional non-linearity in the viscous term to prevent unwanted singularities while advancing the stochastic differential equation.

As a final note, the scaling of $\delta \sim \tau_{\eta}^{-1}$ contradicts the RFD model for the viscous Laplacian, which used the integral timescale and thus introduced a $R e_{\lambda}^{-1}$ scaling for the viscous term. While $R e_{\lambda}$ dependence can be introduced in the present model through the skewness coefficient, the similar difficulties as encountered by the RFD model are seen when going to large Reynolds numbers. It is the authors' view that a fixed skewness coefficient, $\mathcal{S}=-0.6$, is appropriate for the present model's the level of fidelity.

\section{REFERENCES}

Arnèodo, A., Benzi, R., Berg, J., Biferale, L., Bodenschatz, E., Busse, A., Calzavarini, E., Castaing, B., Cencini, M., Chevillard, L., Fisher, R. T., Grauer, R., Homann, H., Lamb, D., Lanotte, A., Lévèque, E., Lüthi, B., Mann, J., Mordant, N., Müller, W.-C., Ott, S., Ouellette, N., Pinton, J.-F., Pope, S., Roux, S., Toschi, F., Xu, H. \& Yeung, P. K. 2008 Universal Intermittent Properties of Particle Trajectories in Highly Turbulent Flows. Physical Review Letters 100 (25), 254504 .

Arora, D., Behr, M. \& Pasquali, M. 2004 A tensor-based measure for estimating blood damage. Artificial Organs 28 (11), 1002-1015.

Ashurst, W. T., Kerstein, A. R., Kerr, R. M. \& Gibson, C. H. 1987 Alignment of vorticity and scalar gradient with strain rate in simulated NavierStokes turbulence. Physics of Fluids 30 (8), 2343-2353.

Bagheri, F., Mitra, D., Perlekar, P. \& Brandt, L. 2012 Statistics of polymer extensions in turbulent channel flow. Physical Review E 86 (5), 056314.

Balkovsky, E., Fouxon, A. \& Lebedev, V. 2000 Turbulent Dynamics of Polymer Solutions. Physical Review Letters 84 (20), 4765-4768.

Batchelor, G. K. 1951 Pressure fluctuations in isotropic turbulence. Mathematical Proceedings of the Cambridge Philosophical Society 47 (2), 359-374.

BAtChELOR, G. K. 1980 Mass transfer from small particles suspended in turbulent fluid. Journal of Fluid Mechanics 98, 609.

Behbahani, M., Behr, M., Hormes, M., Steinseifer, U., Arora, D., Coronado, O. \& PASQUALI, M. 2009 A review of computational fluid dynamics analysis of blood pumps. European Journal of Applied Mathematics 20, 363.

Bershadskit, A., Kit, E. \& Tsinober, A. 1993 On universality of geometrical invariants in turbulenceExperimental results. Physics of Fluids A: Fluid Dynamics 5 (1993), 1523. 
Beтchov, R. 1956 An inequality concerning the production of vorticity in isotropic turbulence. Journal of Fluid Mechanics 1 (05), 497-504.

Biferale, L., Bodenschatz, E., Cencini, M., Lanotte, A. S., Ouellette, N. T., Toschi, F. \& XU, H. 2008 Lagrangian structure functions in turbulence: A quantitative comparison between experiment and direct numerical simulation. Physics of Fluids 20 (6), 065103.

Biferale, L., Boffetta, G., Celani, A., Devenish, B. J., Lanotte, A. \& Toschi, F. 2004 Multifractal Statistics of Lagrangian Velocity and Acceleration in Turbulence. Physical Review Letters 93 (6), 064502.

Biferale, L., Meneveau, C. \& Verzicco, R. 2014 Deformation statistics of sub-Kolmogorovscale ellipsoidal neutrally buoyant drops in isotropic turbulence. Journal of Fluid Mechanics 754, 184-207.

Biferale, L., Scagliarini, A. \& Toschi, F. 2010 On the measurement of vortex filament lifetime statistics in turbulence. Physics of Fluids 22 (6), 065101.

Boffetta, G., De Lillo, F. \& Musacchio, S. 2002 Lagrangian statistics and temporal intermittency in a shell model of turbulence. Physical Review E 66 (6), 066307.

Cantwell, B. J. 1992 Exact solution of a restricted Euler equation for the velocity gradient tensor. Physics of Fluids 4 (4), 782-793.

Chen, H., Chen, S. \& Kraichnan, R. H. 1989 Probability Distribution of a Stochastically Advected Scalar Field. Physical Review Letters 63 (24), 2657-2660.

Chertkov, M. 2000 Polymer Stretching by Turbulence. Physical Review Letters 84 (20), 47614764, arXiv: 9911011.

Chertkov, M., Pumir, A. \& Shraiman, B. I. 1999 Lagrangian tetrad dynamics and the phenomenology of turbulence. Physics of Fluids 11 (8), 2394-2410, arXiv: 9905027.

Chevillard, L. \& Meneveau, C. 2006 Lagrangian Dynamics and Statistical Geometric Structure of Turbulence. Physical Review Letters 97 (17), 174501.

Chevillard, L. \& Meneveau, C. 2011 Lagrangian time correlations of vorticity alignments in isotropic turbulence: Observations and model predictions. Physics of Fluids 23 (10), 101704 .

Chevillard, L. \& Meneveau, C. 2013 Orientation dynamics of small , triaxial-ellipsoidal particles in isotropic turbulence. Journal of Fluid Mechanics 737, 571-596, arXiv: arXiv:1305.6275v1.

Chevillard, L., Meneveau, C., Biferale, L. \& Toschi, F. 2008 Modeling the pressure Hessian and viscous Laplacian in turbulence: Comparisons with direct numerical simulation and implications on velocity gradient dynamics. Physics of Fluids 20 (10), 101504.

Chevillard, L., Roux, S. G., Leveque, E., Mordant, N., Pinton, J. \& Arneodo, A. 2003 Lagrangian Velocity Statistics in Turbulent Flows: Effects of Dissipation. Physical Review Letters 91 (21), 214502.

De Tullio, M. D., Nam, J., Pascazio, G., Balaras, E. \& Verzicco, R. 2012 Computational prediction of mechanical hemolysis in aortic valved prostheses. European Journal of Mechanics, B/Fluids 35, 47-53.

Donzis, D. A., Yeung, P. K. \& Sreenivasan, K. R. 2008 Dissipation and enstrophy in isotropic turbulence: Resolution effects and scaling in direct numerical simulations. Physics of Fluids 20 (4), 045108.

Falkovich, G., Gawedski, K. \& Vergassola, M. 2001 Particles and fields in fluid turbulence. Rev. Mod. Phys. 73 (October), 913-975.

Frisch, U. 1995 Turbulence: The Legacy of A. N. Kolmogorov. Cambridge University Press.

Girimaji, S. S. \& Pope, S. B. 1990 Material-element deformation in isotropic turbulence. Journal of Fluid Mechanics 220, 427-458.

Green, M. A., Rowley, C. W. \& Haller, G. 2007 Detection of Lagrangian coherent structures in three-dimensional turbulence. Journal of Fluid Mechanics 572, 111-120.

HALler, G. 2000 Finding finite-time invariant manifolds in two-dimensional velocity fields. Chaos (Woodbury, N.Y.) 10 (1), 99-108.

Haller, G. 2015 Langrangian Coherent Structures. Annual Review of Fluid Mechanics 47 (1), $137-162$. 
HALleR, G. \& YuAN, G. 2000 Lagrangian coherent structures and mixing in two-dimensional turbulence. Physica D 147 (3-4), 352-370.

JeOng, E. \& Girimaji, S. S. 2003 Velocity-Gradient Dynamics in Turbulence: Effect of Viscosity and Forcing. Theoretical and Computational Fluid Dynamics 16 (6), 421-432.

Karp-Boss, L., Boss, E. \& Jumars, P. A. 1996 Nutrient Fluxes to Planktonic Osmotrophs in the Presence of Fluid Motion. Oceanography and Marine Biology: An Annual Review 34, 71-107.

Kolmogorov, A. N. 1941 The local structure of turbulence in incompressible viscous fluid for very large Reynolds numbers. Dokl. Akad. Nauk SSSR 30, 299-303.

Kolmogorov, A. N. 1962 A refinement of previous hypotheses concerning the local structure of turbulence in a viscous incompressible fluid at high Reynolds number. Journal of Fluid Mechanics 13 (01), 82-85.

Kraichnan, R. H. 1990 Models of Intermittency in Hydrodynamic Turbulence. Physical Review Letters 65 (5), 575-578.

LAWson, J. \& DAWson, J. 2015 On velocity gradient dynamics and turbulent structure. Journal of Fluid Mechanics 780, 60-98.

Li, Y., Perlman, E., Wan, M., Yang, Y., Meneveau, C., Burns, R., Chen, S., Szalay, A. \& EYINK, G. 2008 A public turbulence database cluster and applications to study Lagrangian evolution of velocity increments in turbulence. Journal of Turbulence $\mathbf{9}$ (31), $1-29$.

Lund, T. S. \& Rogers, M. M. 1994 An improved measure of strain state probability in turbulent flows. Physics of Fluids 6 (5), 1838-1847.

Lüthi, B., Holzner, M. \& Tsinober, A. 2009 Expanding the Q? R space to three dimensions. Journal of Fluid Mechanics 641, 497-507.

Maniero, R., Masbernat, O., Climent, E. \& Risso, F. 2012 Modeling and simulation of inertial drop break-up in a turbulent pipe flow downstream of a restriction. International Journal of Multiphase Flow 42, 1-8.

Martin, J., Dopazo, C. \& Valino, L. 1998a Dynamics of velocity gradient invariants in turbulence: Restricted Euler and linear diffusion models. Physics of Fluids 10 (8), 20122025.

Martin, J., Ooi, A., Chong, M. S. \& Soria, J. $1998 b$ Dynamics of the velocity gradient tensor invariants in isotropic turbulence. Physics of Fluids 10, 2336.

Martins-Afonso, M. \& Meneveau, C. 2010 Recent fluid deformation closure for velocity gradient tensor dynamics in turbulence: Timescale effects and expansions. Physica D: Nonlinear Phenomena 239 (14), 1241-1250.

Meneveau, C. 2011 Lagrangian Dynamics and Models of the Velocity Gradient Tensor in Turbulent Flows. Annual Review of Fluid Mechanics 43, 219-245.

Meneveau, C. \& Lund, T. S. 1994 On the Lagrangian nature of the turbulence energy cascade. Physics of Fluids 6 (8), 2820.

Meneveau, C. \& Sreenivasan, K. R. 1991 The multifractal nature of turbulent energy dissipation. Journal of Fluid Mechanics 224, 429-484.

Moriconi, L., Pereira, R. M. \& Grigorio, L. S. 2014 Velocity-gradient probability distribution functions in a lagrangian model of turbulence. Journal of Statistical Mechanics: Theory and Experiment 2014, P10015, arXiv: arXiv:1401.0678v1.

Nomura, K. K. \& Post, G. K. 1998 The structure and dynamics of vorticity and rate of strain in incompressible homogeneous turbulence. Journal of Fluid Mechanics 377, 65-97.

Oвоukнov, A. M. 1962 Some specific features of atmospheric tubulence. Journal of Fluid Mechanics 13 (01), 77-81.

Ohkitani, K. \& Kishiba, S. 1995 Nonlocal nature of vortex stretching in an inviscid fluid. Physics of Fluids $\mathbf{7}$ (2), 411.

Ooi, A., Martin, J., Soria, J. \& Chong, M. S. 1999 A study of the evolution and characteristics of the invariants of the velocity-gradient tensor in isotropic turbulence. Journal of Fluid Mechanics 381, 141-174.

Oтtino, J. M. 1989 The Kinematics of Mixing, Stretching, Chaos, and Transport. Cambridge, UK: Cambridge University Press.

PARISI, G. \& Frisch, U. 1985 On the singularity structure of fully developed turbulence. In 
Turbulence and Predictability in Geophysical Fluid Dynamics (ed. M. Ghil, R. Benzi \& G. Parisi). North-Holland, New York.

Parsa, S., Calzavarini, E., Toschi, F. \& Voth, G. A. 2012 Rotation rate of rods in turbulent fluid flow. Physical Review Letters 109 (September), 1-4, arXiv: arXiv:1205.0219v1.

Pedley, T. J. \& Kessler, J. 1992 Hydrodynamic Phenomena In Suspensions Of Swimming Microorganisms. Annual Review of Fluid Mechanics 24, 313-358.

Perlman, E., Burns, R., Li, Y. \& Meneveau, C. 2007 Data exploration of turbulence simulations using a database cluster. Proceedings of the 2007 ACM/IEEE Conference on Supercomputing (SC'O7) .

Pope, S. B. 1991 Mapping Closures for Turbulent Mixing and Reaction. Theoretical and Computational Fluid Dynamics 2, 255-270.

Pope, S. B. 2000 Turbulent Flows. Cambridge, UK: Cambridge University Press.

Press, W. H., Teukolsky, S. A., Vetterling, W. T. \& Flannery, B. P. 1992 Numerical Recipes in Fortran 77, 2nd edn. New York, NY: Cambridge University Press.

Rosales, C. \& Meneveau, C. 2008 Anomalous scaling and intermittency in three-dimensional synthetic turbulence. Physical Review E - Statistical, Nonlinear, and Soft Matter Physics 78, $1-18$.

Sreenivasan, K. R. \& Antonia, R. A. 1997 The Phenomenology of Small-Scale Turbulence. Annual Review of Fluid Mechanics 29 (1), 435-472.

Suman, S. \& GiRimaji, S. S. 2009 Homogenized Euler equation: a model for compressible velocity gradient dynamics. Journal of Fluid Mechanics 620, 177.

Suman, S. \& GiRIMAJI, S. S. 2011 Dynamical model for velocity-gradient evolution in compressible turbulence. Journal of Fluid Mechanics 683, 289-319.

Toschi, F. \& Bodenschatz, E. 2009 Lagrangian Properties of Particles in Turbulence. Annual Review of Fluid Mechanics 41 (1), 375-404.

Vieillefosse, P. 1982 Local interaction between vorticity and shear in a perfect incompressible fluid. Journal de Physique 43, 837-842.

Vieillefosse, P. 1984 Internal motion of a small element of fluid in an inviscid flow. Physica A: Statistical Mechanics and its Applications 125, 150-162.

WALlaCe, J. M. 2009 Twenty years of experimental and direct numerical simulation access to the velocity gradient tensor: What have we learned about turbulence? Physics of Fluids 21 (2), 021301.

WilczeK, M. \& Meneveau, C. 2014 Pressure Hessian and viscous contributions to velocity gradient statistics based on Gaussian random fields. Journal of Fluid Mechanics 756, $191-225$.

Yu, H. \& Meneveau, C. 2010 Lagrangian Refined Kolmogorov Similarity Hypothesis for Gradient Time Evolution and Correlation in Turbulent Flows. Physical Review Letters $104(8), 084502$. 